\title{
Structural Basis for the C-Terminal Domain of Mycobacterium tuberculosis Ribosome Maturation Factor RimM to Bind Ribosomal Protein S19
}

\author{
Haoran Zhang $\mathbb{D}^{\circ}$, Qiuxiang Zhou ${ }^{\mathbb{C}}$, Chenyun Guo, Liubin Feng, Huilin Wang, Xinli Liao and Donghai Lin * \\ MOE Key Laboratory of Spectrochemical Analysis \& Instrumentation, Key Laboratory of Chemical Biology of \\ Fujian Province, College of Chemistry and Chemical Engineering, Xiamen University, Xiamen 361005, China; \\ hrzhang@outlook.com (H.Z.); Camille_xiang99@163.com (Q.Z.); guochy78@xmu.edu.cn (C.G.); \\ tonyfeng@xmu.edu.cn (L.F.); wanghuilin@stu.xmu.edu.cn (H.W.); xlliao@xmu.edu.cn (X.L.) \\ * Correspondence: dhlin@xmu.edu.cn
}

check for updates

Citation: Zhang, H.; Zhou, Q.; Guo, C.; Feng, L.; Wang, H.; Liao, X.; Lin, D. Structural Basis for the C-Terminal Domain of Mycobacterium tuberculosis Ribosome Maturation Factor RimM to Bind Ribosomal Protein S19. Biomolecules 2021, 11, 597. https:/ / doi.org/10.3390/biom11040597

Academic Editor: Vladimir N. Uversky

Received: 30 March 2021

Accepted: 17 April 2021

Published: 18 April 2021

Publisher's Note: MDPI stays neutral with regard to jurisdictional claims in published maps and institutional affiliations.

Copyright: (c) 2021 by the authors. Licensee MDPI, Basel, Switzerland. This article is an open access article distributed under the terms and conditions of the Creative Commons Attribution (CC BY) license (https:/ / creativecommons.org/licenses/by/ $4.0 /)$.

\begin{abstract}
Multidrug-resistant tuberculosis (TB) is a serious threat to public health, calling for the development of new anti-TB drugs. Chaperon protein RimM, involved in the assembly of ribosomal protein S19 into 30S ribosomal subunit during ribosome maturation, is a potential drug target for TB treatment. The C-terminal domain (CTD) of RimM is primarily responsible for binding S19. However, both the CTD structure of RimM from Mycobacterium tuberculosis (MtbRimM $\mathrm{CTD}_{\text {) }}$ ) and the molecular mechanisms underlying $M t b$ Rim $_{\text {CTD }}$ binding $\mathrm{S} 19$ remain elusive. Here, we report the solution structure, dynamics features of $M t b \operatorname{RimM}_{\mathrm{CTD}}$, and its interaction with $\mathrm{S} 19 . M t b \operatorname{Rim} \mathrm{M}_{\mathrm{CTD}}$ has a rigid hydrophobic core comprised of a relatively conservative six-strand $\beta$-barrel, tailed with a short $\alpha$-helix and interspersed with flexible loops. Using several biophysical techniques including surface plasmon resonance (SPR) affinity assays, nuclear magnetic resonance (NMR) assays, and molecular docking, we established a structural model of the $M t b \mathrm{RimM}_{\mathrm{CTD}}-\mathrm{S} 19$ complex and indicated that the $\beta 4-\beta 5$ loop and two nonconserved key residues (D105 and H129) significantly contributed to the unique pattern of $M t b$ RimM $\mathrm{CTD}_{\mathrm{C}}$ binding S19, which might be implicated in a form of orthogonality for species-dependent RimM-S19 interaction. Our study provides the structural basis for MtbRimM $\mathrm{CTD}$ binding S19 and is beneficial to the further exploration of $M t b \operatorname{RimM}$ as a potential target for the development of new anti-TB drugs.
\end{abstract}

Keywords: protein structure; Mycobacterium tuberculosis; ribosome maturation factor RimM; NMR spectroscopy; protein dynamics; MD simulation; protein-protein docking

\section{Introduction}

As a deadly infectious disease, tuberculosis (TB) infected about 10 million people and caused an estimated 1.4 million deaths worldwide in 2019, and the responsible pathogen for TB is Mycobacterium tuberculosis (Mtb) [1]. Globally, TB has developed resistance to traditional anti-TB drugs like isoniazid and rifampicin, an unfortunate complication to TB prevention and treatment [2,3]. Patients infected with multidrug-resistant TB (MDR-TB) require medicines at higher costs and a longer time for treatment, only receiving a treatment success rate of $57 \%$ [1]. With the emergence and spread of MDR-TB strains, it is imminent to find clinical targets for developing new antimicrobials against $M t b$.

RimM, an important ribosome maturation factor protein existing in $M t b$, is a candidate target for anti-TB drugs. The RimM protein family is included in various bacterial species, but no ortholog is available in humans. Researches have been extensively conducted on explicit functions of RimM serving as one of bacterial biogenesis factors active in the ribosome assembly process [4]. Knockout of the RimM gene caused a significant decrease in cell growth rate, accumulation of $16 \mathrm{~S}$ rRNA precursors and ribosomal intermediates, and a reduction of polysome level [5-8]. Further researches showed that RimM does not 
bind to complete $70 \mathrm{~S}$ ribosomes or mature $30 \mathrm{~S}$ subunits but moderately binds to immature $30 \mathrm{~S}$ intermediates $[4,5,9]$. Suppressive mutation experiments on ribosomal proteins and $16 \mathrm{~S}$ rRNA indicated that RimM might bind the $3^{\prime}$-domain of $16 \mathrm{~S}$ rRNA [5,8]. Analyses on composition and structures of the immature 30S intermediates collected in the RimM knockout strain indicated that RimM plays a crucial role in assisting the late assembly of the head domain of the $30 \mathrm{~S}$ subunit $[4,10,11]$. Moreover, in vitro pull-down experiments confirmed the specificity with which RimM binds to S19, a ribosomal protein located at the $30 \mathrm{~S}$ head domain [8]. Kinetic experiments disclosed that RimM accelerates the binding of S19 to $16 \mathrm{~S}$ rRNA by overcoming the potential well during the slow binding process [12]. Together, these advances indicate that the RimM-S19 interaction plays a vital role in ribosome biosynthesis, for which RimM is a competent attacking target for antimicrobials against $M t b$.

As is well known, the function of a protein is determined by its structure, and structural basis is required to mechanistically understand the protein function. So far, the three-dimensional (3D) structure of RimM from $M t b$ (MtbRimM) has not been determined. To our knowledge, the following 3D structures of RimM orthologs have been resolved and are accessible in the Protein Data Bank (PDB): (a) the crystal structures of RimM proteins in free form from P. aeruginosa (3 mutations, PDB ID: 2F1L, termed PaeRimM), T. thermophilus HB8 (wild type, PDB ID: 2DYI, termed TthRimM), A. calcoaceticus (5 mutations, PDB ID: 2QGG, termed AciRimM), and H. influenzae (wild type, PDB ID: 3H9N, termed HinRimM); (b) the crystal structure of the RimM-S19 complex from T. thermophilus HB8 (both wild types, PDB ID: 3A1P, S19 from T. thermophilus is termed TthS19); (c) the solution structure of truncated RimM N-terminal domain from T. thermophilus HB8 (wild type, PDB ID: 2DOG) [13]. Inspection of these structures, in correspondence to earlier assertions based on multi-sequence alignments $[8,14]$, shows that the structure of RimM is composed of an N-terminal domain (NTD), a C-terminal domain (CTD), and a short loop in between (Figure 1A). Part of the RimM NTD shares sequence similarity with the RNA-binding KH domain, implying its potential interaction with RNA [5,15]. The RimM CTD was identified as a PRC-barrel domain [14]. Originally, the PRC-barrels were discovered to be a mediator of quinone reduction within the photosynthetic reaction center complex from purple proteobacteria [16]. However, PRC-barrels of the RimM family lack a decisive glutamate residue required for electron transfer in the redox reaction [14]. Instead, they are likely to be involved in binding S19 during the maturation of the 30S ribosomal subunit [8]. Previous nuclear magnetic resonance (NMR) titration assays showed that the CTD part of full-length RimM can significantly interact with S19 but the NTD part can not, indicating that the CTD part is primarily responsible for binding S19 [13].

Suffering from very few available conformational restraints, the structural calculation of TthRimM CTD failed to converge in the NMR structure ensemble, in sharp contrast with TthRimM NTD [13]. On the other hand, the aforementioned crystal structures of full-length RimM orthologs characterize a well-folded CTD, and the TthRimM-S19 complex even describes a molecular model for the RimM-S19 interaction. However, sequence alignments show that the highest sequence identity between MtbRimM CTD (termed MtbimM ${ }_{\text {CTD }}$ ) and other CTDs from species with known structures is only 32.48\% (Figure 1B). Thus, the orthologous structures of RimM are insufficient for revealing the structural basis of $M t b$ RimM ${ }_{\mathrm{CTD}}$ binding S19. Expectedly, such a structural basis would facilitate the design of RimM-based anti-TB drugs.

Here, we determined the solution structure of $M t b \operatorname{RimM}_{\mathrm{CTD}}$ that is primarily responsible for the interaction of MtbRimM with S19. We then analyzed dynamics features of $M t b R_{\text {RimM }}$ CTD by NMR relaxation measurements and molecular dynamics (MD) simulation, and characterized biophysical properties of $M t b \operatorname{RimM}_{\mathrm{CTD}}$ binding S19 through NMR titration and surface plasmon resonance (SPR) assays. Based on the identified S19 binding sites on $M t b \operatorname{RimM}_{\mathrm{CTD}}$, which were verified by mutagenesis experiments, we established a structural model of the $M t b R i m M_{C T D}-S 19$ complex by molecular docking to illustrate the unique pattern of $M t b$ RimM $M_{C T D}$ binding S19. Our results shed light on the molecular mech- 
anisms of the MtbRimM-S19 interaction and offer novel insights into drug development against tuberculosis.

A

Full-length MtbRimM

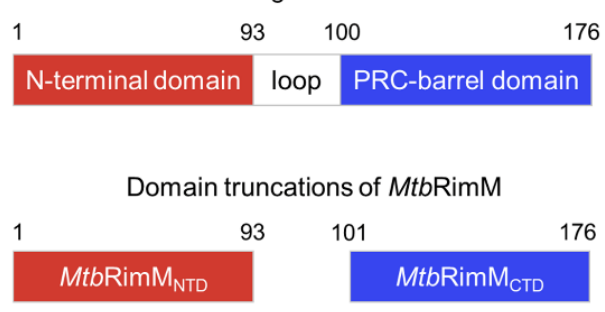

B
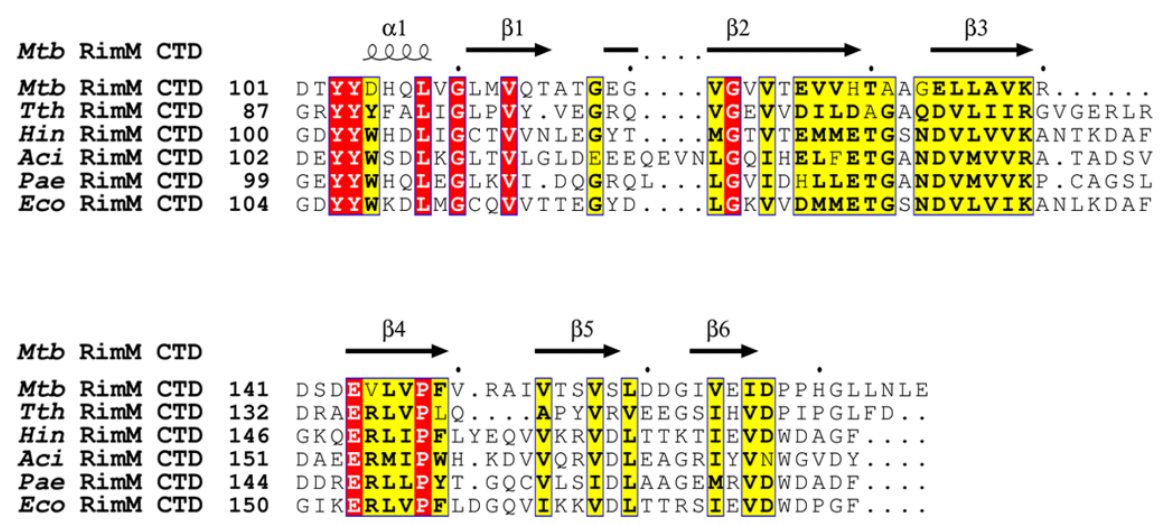

Figure 1. RimM consists of the N-terminal domain (NTD) and C-terminal domain (CTD). (A) Schematic diagram of two domains in RimM from Mycobacterium Tuberculosis (MtbRimM) predicted by Pfam. (B) Sequence alignments between CTDs of MtbRimM and RimM orthologs. Identical residues are highlighted in red and similar residues in yellow, and secondary structure elements of the CTD of $M t b \operatorname{RimM}\left(M t b\right.$ RimM $_{\mathrm{CTD}}$ ) resolved in this study (see Section 3.1) are shown above the alignments.

\section{Materials and Methods}

\subsection{Cloning, Expression, and Purification}

The gene sequences of RimM and S19 from M. tuberculosis strain H37Rv (MtbRimM and MtbS19) were obtained from the NCBI database (gene ID: 887188 for RimM and 888356 for S19). Recombinant plasmids harboring MtbRimM gene (pET-22b, with a C-terminal His $_{6}{ }^{-}$ tag) or MtbS19 gene (pET-28a, with thrombin-cleavable N-terminal His 6 -tag) were commercially synthesized (GenScript, Nanjing, China) Boundaries for the N-terminal domain (residues 4-93, NTD) and the C-terminal domain (residues 100-173, CTD) of MtbRimM were determined by Pfam [17]. To obtain truncations, individual gene fragments of the NTD of $M t b \operatorname{RimM}\left(M t b \operatorname{RimM}_{\mathrm{NTD}}\right.$, residues 1-93) and the CTD of $M t b \operatorname{RimM}\left(M t b \operatorname{RimM} \mathrm{CTD}_{\mathrm{C}}\right.$, residues 101-176) were extracted and cloned by PCR. The following primers were applied in the PCR: (a) 5'-TTAGGATCCATGGAGCTGGTTGTGG-3' (restriction site BamH I) and (b) 5'-TAGCTCGAGTTAATCGTCCGCATCG-3' (restriction site Xho I) for MtbRimM ${ }_{\mathrm{NTD}}$, and (c) 5'-CGCCGGCATATGGATACCTACTATG-3' (restriction site Nde I) and (d) 5'TAGCTCGAGTTCCAGGTTCAGCAGA-3' (restriction site Xho I) for MtbRimM ${ }_{\text {CTD. PCR }}$ products for $M t b$ RimM $M_{N T D}$ were then ligated into the pET-28a-SUMO plasmid which contains an N-terminal $\mathrm{His}_{6}$-tag followed by a SUMO fusion protein and a SUMO protease cutting site [18], and those for $M t b$ RimM ${ }_{C T D}$ into the $\mathrm{pET}-22 \mathrm{~b}$ plasmid which contains a C-terminal His $_{6}$-tag. Recombinant plasmids encoding $M t b$ RimM $_{\mathrm{CTD}}$ point mutants (pET22b, with C-terminal $\mathrm{His}_{6}$-tag) were commercially synthesized (Sangon Biotech, Shanghai, China). All plasmids used in this study were verified via DNA sequencing. 
The plasmids bearing the genes of $M t b \operatorname{RimM}, M t b S 19, M t b \operatorname{RimM}_{\mathrm{NTD}}, M t b \operatorname{Rim} \mathrm{M}_{\mathrm{CTD}}$ and point mutants were transformed into the E. Coli BL21(DE3) strain. Overexpression of these proteins was induced with $0.5 \mathrm{mM}$ IPTG at $\mathrm{OD}_{600}=0.6$ and conducted at $25{ }^{\circ} \mathrm{C}$ for $10 \mathrm{~h}$ in either LB liquid media or M9 media. For uniform ${ }^{15} \mathrm{~N}$ - and $/$ or ${ }^{13} \mathrm{C}$-labeling of the proteins, $0.1 \%(\mathrm{~m} / \mathrm{v})$ of ${ }^{15} \mathrm{NH}_{4} \mathrm{Cl}$ and/or $0.3 \%(\mathrm{~m} / \mathrm{v})$ of ${ }^{13} \mathrm{C}$-glucose were added into M9 media.

The harvested cell pellet was resuspended in $50 \mathrm{mM}$ Tris, $\mathrm{pH} 8.0,950 \mathrm{mM} \mathrm{NaCl}$, $2.0 \mathrm{mM}$ imidazole, $0.1 \mathrm{mg} / \mathrm{mL}$ lysozyme, $1.0 \mathrm{mM}$ phenylmethylsulfonyl fluoride (PMSF), and lysed on ice by sonication. The soluble fraction of the lysate was collected by centrifugation and loaded onto $5 \mathrm{~mL}$ Co-NTA resin, which was obtained by stripping the Ni-NTA resin (GE Healthcare Bio-Sciences AB, Uppsala, Sweden) of coordinated $\mathrm{Ni}^{2+}$ with EDTA and re-cobaltizing it with $\mathrm{CoCl}_{2}$. In the affinity chromatography, recombinant protein was eluted with $50 \mathrm{mM}$ Tris, $\mathrm{pH} 8.0,250 \mathrm{mM} \mathrm{NaCl}, 300 \mathrm{mM}$ imidazole. After that, the protein was buffer-exchanged into $20 \mathrm{mM}$ potassium phosphate, $\mathrm{pH} 7.2,100 \mathrm{mM} \mathrm{KCl}, 0.02 \%$ $\mathrm{NaN}_{3}$ (hereafter referred to as the universal buffer), and purified through size exclusion chromatography (SEC) using ÄKTA FPLC system with a Superdex 75 10/300 GL column (GE healthcare). Additionally, proteins with cleavable $\mathrm{N}$-terminal $\mathrm{His}_{6}$-tags were treated with corresponding proteases until the tags were completely cleaved before repurified with Co-NTA and SEC. MtbRimM NTD was cleaved with $0.4 \mathrm{mg} / \mathrm{mL}$ SUMO-protease for $3 \mathrm{~h}$ at room temperature, and $M t b S 19$ with $0.4 \mathrm{mg} / \mathrm{mL}$ thrombin overnight at $4{ }^{\circ} \mathrm{C}$. Finally, the protein solution was concentrated to $600 \mu \mathrm{M}$ for NMR experiments conducted in this study if not otherwise specified.

\subsection{NMR Spectroscopy}

NMR spectra were recorded at $298 \mathrm{~K}$ on a Bruker Avance III $850 \mathrm{MHz}$ spectrometer (Bruker BioSpin GmbH, Karlsruhe, Germany) equipped with a ${ }^{1} \mathrm{H}-/{ }^{13} \mathrm{C}-/{ }^{15} \mathrm{~N}$ - TCI cryogenic probe (Bruker AG, Fällanden, Switzerland). All protein samples for NMR spectroscopy were dissolved in the universal buffer. The protein samples included $10 \%$ $\mathrm{D}_{2} \mathrm{O}(v / v)$ for recording general NMR spectra and $99 \% \mathrm{D}_{2} \mathrm{O}(v / v)$ for recording $3 \mathrm{D}^{13} \mathrm{C}$ edited NOESY-HSQC (nuclear Overhauser effect spectroscopy-heteronuclear singular quantum correlation) spectrum. The $\left[{ }^{15} \mathrm{~N}\right]$-labeled protein sample was used for recording two-dimensional (2D) ${ }^{1} \mathrm{H}_{-}{ }^{15} \mathrm{~N}$ HSQC and 3D ${ }^{15} \mathrm{~N}$-edited NOESY-HSQC spectra, and the $\left[{ }^{13} \mathrm{C},{ }^{15} \mathrm{~N}\right]$-labeled protein sample was prepared for $2 \mathrm{D}{ }^{1} \mathrm{H}-{ }^{13} \mathrm{C}$ HSQC and other $3 \mathrm{D}$ NMR spectra. 2D ${ }^{1} \mathrm{H}_{-}{ }^{15} \mathrm{~N}$ HSQC and ${ }^{1} \mathrm{H}_{-}{ }^{13} \mathrm{C}$ HSQC spectra were recorded on $\mathrm{Mtb}$ RimM, $M t b \operatorname{Rim} M_{N T D}$ and $M t b$ RimM ${ }_{\text {CTD }}$. 3D HNCACB, CBCA(CO)NH, HNCA, HN(CO)CA, $\mathrm{HNCO}$ and $\mathrm{HN}(\mathrm{CA}) \mathrm{CO}$ spectra were recorded for performing backbone resonance assignments of $M t b \operatorname{RimM}$ and $M t b \operatorname{RimM}_{\mathrm{CTD}}$. 3D H(CCO)NH, CC(CO)NH, HBHA(CO)NH, $\mathrm{H}(\mathrm{C}) \mathrm{CH}-\mathrm{COSY}$, and $(\mathrm{H}) \mathrm{CCH}-\mathrm{TOCSY}$ spectra were recorded for conducting side-chain resonance assignments of $M t b$ RimM $M_{C T D} .3 D^{13} \mathrm{C}$-edited NOESY-HSQC and ${ }^{15} \mathrm{~N}$-edited NOESY-HSQC spectra with a mixing time of $120 \mathrm{~ms}$ were recorded on $M t b$ Rim $\mathrm{M}_{\mathrm{CTD}}$ for obtaining NOE (nuclear Overhauser effect) restraints. All spectra were processed with NMRPipe [19] and analyzed with NMRFAM-SPARKY [20].

\subsection{Structure Determination}

Chemical shifts of $M t b$ RimM $M_{C T D}$ which had been previously deposited to BMRB (Accession ID: 36368) were used for NOE assignments (data under review) [21]. Cross-peaks in ${ }^{13} \mathrm{C}$ - and ${ }^{15} \mathrm{~N}$-edited NOESY-HSQC spectra were either manually assigned or ambiguously assigned with the help of Aria 2.3 [22]. NOE-derived distance restraints were generated from signal integrals in ${ }^{13} \mathrm{C}$ - and ${ }^{15} \mathrm{~N}$-edited NOESY-HSQC spectra. Backbone dihedral restraints $(\varphi, \psi)$ were predicted from chemical shifts via the TALOS-N server [23]. Altered MD parameters were adopted in Aria 2.3 setup (10,000, 4000, 80,000, and 64,000 for high temperature, refinement, cool1, and cool2 steps). Totally, 100 structures of $M t b$ RimM $M_{C T D}$ were calculated and refined with Aria 2.3, and 20 lowest-energy models were used as the ultimate structure ensemble. The C-terminal $\mathrm{His}_{6}$-tag was not modeled in the final structures 
due to the lack of chemical shifts and NOE cross-peak assignments. PROCHECK [24] was applied for structural quality evaluation, and Pymol [25] for structure visualization.

\subsection{NMR Relaxation Measurements}

All recombinant proteins were dissolved in the universal buffer. A protein sample of $\left[{ }^{15} \mathrm{~N}\right]$-labeled $M t b$ RimM ${ }_{\mathrm{CTD}}$ at $600 \mu \mathrm{M}$ was used to conduct NMR relaxation measurements of backbone amide groups including amide $\mathrm{R}_{1}$ and $\mathrm{R}_{2}$ relaxation rates and $\left\{{ }^{1} \mathrm{H}\right\}{ }^{15} \mathrm{~N}$ heteronuclear steady-state NOEs (hNOEs). Two sets of pseudo-3D experiments incorporated with ${ }^{1} \mathrm{H}-{ }^{15} \mathrm{~N}$ HSQC spectra were recorded at $298 \mathrm{~K}, 850 \mathrm{MHz}$ for $\mathrm{R}_{1}$ and $\mathrm{R}_{2}$ measurements, and repeated $2 \mathrm{D} \mathrm{hNOE} \mathrm{spectra} \mathrm{were} \mathrm{recorded} \mathrm{at} \mathrm{the} \mathrm{same} \mathrm{condition} \mathrm{for} \mathrm{hNOE} \mathrm{measure-}$ ments and error analysis. $R_{1}$ values were calculated with relaxation delays of $10,50,100$ $(\times 2), 200,400,600,800(\times 2), 1200,1600$, and $2000 \mathrm{~ms}$, while $R_{2}$ values were determined with relaxation delays of $16.32,32.64(\times 2), 48.96,65.28,81.60,97.92,114.24,130.56(\times 2)$, 146.88 , and $163.20 \mathrm{~ms}$. The hNOEs were obtained in interleaved spectra with and without a 3-s ${ }^{1} \mathrm{H}$ pre-saturation, the latter being replaced by a 3-s relaxation delay. Peak intensities were represented by peak heights for data analysis. NMRFAM-SPARKY [20] was used to fit exponential decay curves to the experimental serial data for determining $R_{1}$ and $R_{2}$ rates, where standard errors of rate constants were estimated by the spread in five repeated Gaussian distribution fits for random noise perturbing peak heights. Residues 131, 151, and 154 were subjugated to signal overlapping or broadening, thus unavailable for spin relaxation analysis. Therefore, a total of 71 residues were analyzed to access backbone dynamics, with prolines, the starting methionine, and the C-terminal $\mathrm{His}_{6}$-tag excluded.

\subsection{Model-Free Analysis}

The FAST-Modelfree program (Version 1.3, Loria Lab, New Haven, CT, USA) [26] was applied to extract dynamics parameters $\left(S^{2}, \tau_{e}, R_{e x}\right)$ based on relaxation data of $M t b R i m M_{C T D}$, which used estimated initial tensors of the protein as input. The Tensor 2 program was employed to estimate the initial tensors [27]. Spin diffusion tensors were then iteratively optimized under an axially-symmetric model. The closest-to-average model in the $M t b R i m M_{C T D}$ structure ensemble (hereafter referred to as the $M t b R i m M_{C T D}$ representative structure) determined in this work was prepared as the structure input for both Tensor 2 and FAST-Modelfree automation. The values of grid-search steps and convergence limit in the FAST-Modelfree setup were 15 and 0.001 for each tensor, respectively. The $S^{2}$ cutoff was set to 0.4 for an all-encompassing characterization of residue spins.

\subsection{NMR Titration Assays}

All recombinant proteins were dissolved in the universal buffer. Either $500 \mu \mathrm{M}\left[{ }^{15} \mathrm{~N}\right]-$ labeled $M t b$ RimM $_{\text {NTD }}$ or $290 \mu \mathrm{M}\left[{ }^{15} \mathrm{~N}\right]$-labeled $M t b \operatorname{RimM} \mathrm{CTD}_{\mathrm{CD}}$ was titrated with unlabeled $M t b S 19$ to a respectively equimolar ratio. The titration of $M t b \mathrm{RimM}_{\mathrm{CTD}}$ into $M t b S 19$ yielded precipitation, a factor unfavorable for a titration assay with higher protein concentrations. For mutual equimolar titrations between $M t b \operatorname{Rim} M_{N T D}$ and $M t b R i m M_{C T D}$, both proteins at a concentration of $150 \mu \mathrm{M}$ were used. ${ }^{1} \mathrm{H}^{-15} \mathrm{~N}$ HSQC spectra were recorded at each titration point at $298 \mathrm{~K}$. The chemical shift perturbation (CSP) was determined with an empirical formula [28] as

$$
\Delta \delta=\sqrt{\frac{1}{2}\left[\left(\Delta \delta_{H}\right)^{2}+0.14\left(\Delta \delta_{N}\right)^{2}\right]}
$$

in which $\Delta \delta_{H}$ and $\Delta \delta_{N}$ represented chemical shift displacements for ${ }^{1} \mathrm{H}$ and ${ }^{15} \mathrm{~N}$ nuclei observed upon titrations, respectively.

\subsection{Sequence Alignments}

Structure-based sequence alignments among the CTDs of RimM orthologs or among S19 orthologs were generated by Clustal X2 [29]. Visualization of the alignments was performed with ESPript 3.0 [30], where the secondary structure information was extracted by the STRIDE webserver using the determined PDB coordinates of $M t b \operatorname{RimM}_{\mathrm{CTD}}$ [31]. 


\subsection{Simulation}

Molecular dynamics simulation was executed under the ff19SB force field [32] integrated into the AmberTools20 suite [33]. The representative structure of $M t b \mathrm{RimM}_{\mathrm{CTD}}$ was cleaned up with the pdb4amber subroutine before the protein was solvated in an OPC water box [34] extending $10.0 \AA$ from the protein surface. Ten $\mathrm{Na}^{+}$ions were added to the system to neutralize the net charge of $M t b$ RimM $_{\mathrm{CTD}}$. A two-stage energy minimization was performed to discard bad contacts: first, the water molecules alone; next, the entire system. After that, a three-stage system equilibration totaling 300 ps was conducted: the system was heated from 0 to $300 \mathrm{~K}$ under NVT ensemble for $100 \mathrm{ps}$ and was run at $300 \mathrm{~K}$ under NVT and NPT ensembles successively for a respective 100 ps. Subsequently, a 120-ns MD simulation under NPT ensemble at $300 \mathrm{~K}$ was performed. Langevin dynamics was adopted for temperature regulation with a collision frequency of $1 \mathrm{ps}^{-1}$ and a time-based pseudorandom seed. Hydrogen-involving bonds were constrained by the SHAKE algorithm [35] and omitted for force evaluation. The nonbonded cutoff was specified as $12 \AA$.

The cpptraj subroutine [36] incorporated within AmberTools20 was utilized for MD data extraction and analysis. Root mean square fluctuation (RMSF) per residue throughout the simulation was calculated with reference to the representative structure of $M t b R i m M_{C T D}$, to which the structures in all frames were RMS-fitted prior to calculation. Secondary structures were determined using the built-in DSSP engine [37].

\subsection{Molecular Docking}

Due to possible backbone conformational changes upon $M t b \operatorname{RimM}_{\mathrm{CTD}}-\mathrm{S} 19$ binding, molecular docking with backbone flexibility [38] was carried out via RosettaDock 4.0 (Rosetta Commons) [39]. As the two starting structures, the representative structure of $M t b$ RimM $\mathrm{CTD}_{\mathrm{CTD}}$ (termed $\mathrm{dA}$ ) was chosen, and the structural model of MtbS19 (termed dB) was built by homology modeling via the SWISS-MODEL server [40], using the crystal structure of S19 in RimM-complexed form from T. thermophilus HB8 (PDB: 3A1P) as the modeling template. The binding interface identified from chemical shift mapping described in this work was considered to engender a rough initial model (termed $\mathrm{dC}$ ) containing both $\mathrm{dA}$ and $\mathrm{dB}$. Totally, 100 conformational ensembles of each protein (termed $\mathrm{dA}^{\prime}$ and $\mathrm{dB}^{\prime}$, respectively) for backbone-flexible docking were generated using $\mathrm{dA}$ and $\mathrm{dB}$ under the unconstrained relax protocol. $\mathrm{dC}$ was also relaxed to spawn a clash-relieved model (termed $\mathrm{dC}^{\prime}$ ) after local refinement. Then, the prepack protocol was run to optimize sidechain rotamers. Finally, with $\mathrm{dA}+\mathrm{dA}^{\prime}$ and $\mathrm{dB}+\mathrm{dB}^{\prime}$ as ensemble candidates for backbone switch and $\mathrm{dC}^{\prime}$ as the initial input structure, unconstrained backbone-flexible docking of MtbRimM $\mathrm{CTD}_{\mathrm{C}}-\mathrm{S} 19$ was performed and 30,000 docking models were calculated. The docking results were evaluated based on the RosettaDock interface energy score. In addition, the method developed by Kumar et al. can be applied to select a particular model out of a large number of docked models, which uses certain constraints for the docking and is then based on the lowest energy plot [41]. Random perturbation subjugated to Gaussian distribution was applied to the input structure prior to every individual simulation, with standard deviations of $3 \AA$ for translation and $8^{\circ}$ for rotation.

\subsection{SPR Affinity Assays}

All recombinant proteins were dissolved in $20 \mathrm{mM}$ potassium phosphate, $\mathrm{pH}$ 7.2, $100 \mathrm{mM} \mathrm{KCl}$ (also used as the system running buffer) for SPR affinity assays. All experiments were performed at $298 \mathrm{~K}$ on a Biacore T200 instrument (GE Healthcare Bio-Sciences $A B$, Uppsala, Sweden). The sandwich approach was employed for the SPR assays: first, the anti-histidine antibody from the His Capture Kit (Cytiva Sweden AB, Uppsala, Sweden) was immobilized to the active surface of a CM5 sensor chip (Cytiva Sweden AB, Uppsala, Sweden) until saturation; second, excessive C-terminal His ${ }_{6}$-tagged $M t b$ RimM $_{\mathrm{CTD}}$ or its mutants flowed through and were captured by the anti-histidine antibody; last, a serial concentration of $M t b S 19$ was injected into the system and captured by $M t b R i m M_{C T D}$. The control surface was treated in the same way, except that MtbS19 solution was substituted 
by blank running buffer. The following concentrations of MtbS19 were used for obtaining SPR assay curves: $0,0.25,0.50,1.0,2.0,4.0$, and $8.0 \mu \mathrm{M}$. Dissociation constants $\left(\mathrm{K}_{\mathrm{D}}\right)$ were determined by fitting the SPR assay curves to the steady-state model.

\section{Results}

\subsection{Solution Structure of MtbRimM $M_{C T D}$}

We first determined the sequential boundaries of NTD and CTD within MtbRimM for the structural determination of $M t b$ Rim $M_{C T D}$. The prediction using Pfam [17] showed that the NTD of $M t b$ RimM covered residues 4-93, the CTD spanned residues 100-173, and in between lay a flexible loop as a linker (Figure 1A). Considering that residue P100 is nonconserved and too hydrophobic as a terminal residue, we omitted P100 in our CTD truncation design to maintain protein solubility. Consequently, we designed recombinant truncations for the NTD (residues 1-93, termed $M t b \operatorname{RimM}_{\mathrm{NTD}}$ ) and the CTD (residues 101-176, termed $M t b$ RimM $\left.{ }_{\mathrm{CTD}}\right)$, and prepared protein samples for further studies.

Chemical shifts for $M t b$ RimM ${ }_{C T D}$ with $99 \%$ backbone ${ }^{15} \mathrm{~N}-{ }^{1} \mathrm{H}$ resonances and $85 \%$ side-chain aliphatic ${ }^{1} \mathrm{H} /{ }^{13} \mathrm{C}$ resonances assigned were previously deposited to BMRB (accession ID: 36368), and ${ }^{13} \mathrm{C}$-edited and ${ }^{15} \mathrm{~N}$-edited NOESY-HSQC spectra were also recorded (under review) [21]. Based on these data, we obtained NOE distance restraints and predicted dihedral angle restraints to calculate the solution structure of $M t b$ RimM $M_{C T D}$. We determined a $M t b$ Rim $M_{\mathrm{CTD}}$ ensemble of 20 lowest-energy models and submitted it to Protein Data Bank (PDB ID: 7CQ1). The NMR restraints and structural statistics are listed in Table 1. The root-mean-squared deviation (RMSD) of backbone atomic coordinates of the ordered region (residues 103-173) to the mean structure reached $0.23 \AA$, indicative of a well-defined structure ensemble. $M t b$ RimM ${ }_{\mathrm{CTD}}$ adopted a $\beta$-barrel structure containing a short $\alpha$-helix ( $\alpha 1$ :D105-L108) and a $\beta$-sheet composed of six strands ( $\beta 1$ : L111-T115, $\beta 2$ : E119-H129, $\beta 3: E 134-K 139, \beta 4: E 144-F 149, \beta 5: V 154-S 158, \beta 6: I 163-I 166)$ in both anti-parallel $(\beta 1-\beta 2-\beta 3-\beta 4, \beta 5-\beta 6)$ and parallel orientations $(\beta 6-\beta 1)$, spatially arranged in the order of $\beta 5-\beta 6-\beta 1-\beta 2-\beta 3-\beta 4$ (Figure $2 \mathrm{~A}$ ). The short $\alpha 1$ helix was situated on the top of the $\beta$-barrel.

Table 1. NMR restraints and structural statistics for $M t b R i m M_{C T D}$.

\begin{tabular}{|c|c|c|}
\hline \multicolumn{2}{|c|}{ NMR Distance and Dihedral Angle Constraints } & Values \\
\hline \multicolumn{2}{|c|}{ Total ambiguous distance restraints } & 117 \\
\hline \multirow{6}{*}{ Unambiguous distance restraints } & Intra-residual & 413 \\
\hline & Sequential $(|i-j|=1)$ & 226 \\
\hline & Short range $(2 \leq|\mathrm{i}-\mathrm{j}| \leq 3)$ & 80 \\
\hline & Medium range $(4 \leq|\mathrm{i}-\mathrm{j}| \leq 5)$ & 50 \\
\hline & Long range $(|\bar{i}-j|>5)$ & 200 \\
\hline & Total & 969 \\
\hline \multirow{3}{*}{$\begin{array}{l}\text { Dihedral angle } \\
\text { restraints }\end{array}$} & $\varphi$ & 63 \\
\hline & $\psi$ & 63 \\
\hline & Total & 126 \\
\hline \multicolumn{3}{|c|}{ Structural Statistics } \\
\hline \multirow{2}{*}{$\begin{array}{c}\text { Mean restraint } \\
\text { violations }\end{array}$} & Distance restraint violations (>0.3 $\mathrm{A})$ & 0 \\
\hline & Dihedral restraint violations $\left(>5^{\circ}\right)$ & 0 \\
\hline \multirow{2}{*}{$\begin{array}{l}\text { Average root-mean-squared-deviation } \\
(\mathrm{RMSD})(\AA) \text { to mean structure (residues } \\
103-173)\end{array}$} & Backbone RMSD & $0.23 \pm 0.05$ \\
\hline & Heavy atoms RMSD & $0.85 \pm 0.10$ \\
\hline \multirow{3}{*}{$\begin{array}{l}\text { Ramachandran } \\
\text { plot statistics }^{1}\end{array}$} & Residues in favored regions & $85.0 \% \pm 2.0 \%$ \\
\hline & Residues in allowed regions & $14.0 \% \pm 2.0 \%$ \\
\hline & Residues in disallowed regions & $1.0 \% \pm 1.0 \%$ \\
\hline
\end{tabular}

\footnotetext{
${ }^{1}$ Accessed from PDB structure validation report.
} 


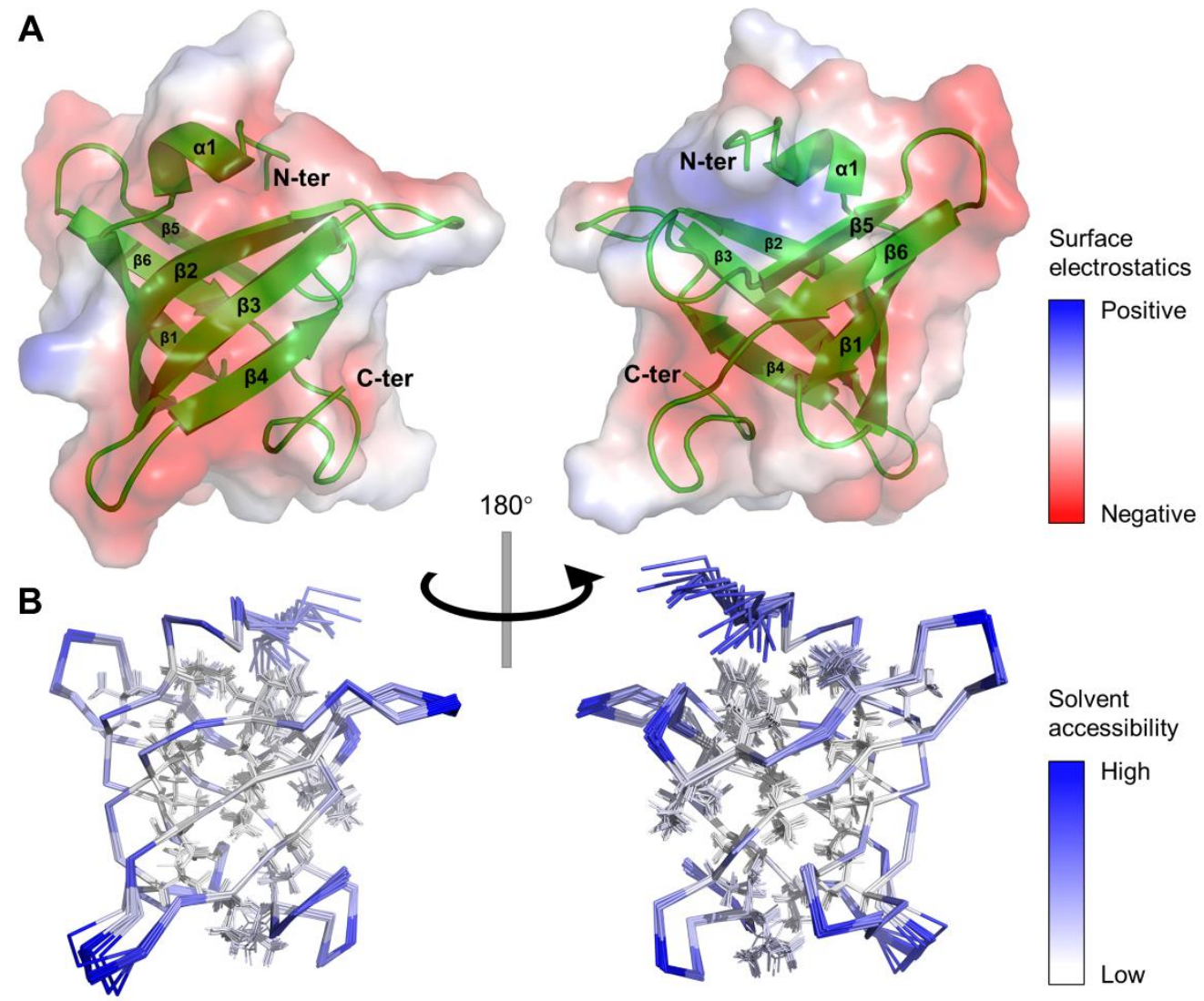

Figure 2. Three-dimensional structure of $M t b \operatorname{Rim} M_{\mathrm{CTD}}$ in solution. (A) Cartoon depiction and surface electrostatics display for the mean structure of $M t b \operatorname{Rim}_{\mathrm{CTD}}$. Positive and negative charges are colored in blue and red on the protein surface with 50\% transparency, respectively. (B) Ribbon depiction of 20 lowest-energy models for $M t b$ RimM $\mathrm{CTD}_{\mathrm{C}}$. Relative solvent accessibility per residue is colored from white to blue in ascending order. Particularly, side chains of non-polar residues buried in the hydrophobic core are presented as lines.

It is noteworthy that most residues on the $\beta$-strands with side chains facing inward were hydrophobic residues, thus forming a hydrophobic core (Figure 2B). Meanwhile, side chains of most charged or polar residues were oriented toward the outer side of the $\beta$-barrel, permitting direct polar contacts to water molecules. The dispersion of positive surface charges was somewhat scattered, while negatively charged residues clustered into larger blocks (Figure 2A). Considering the role of CTD as the S19 binder, these extended negatively charged blocks potentially enabled the binding of positively charged ligands/residues. Non-polar residues that formed the hydrophobic core shared high similarities among species (Figure 1B), implying the need for a stabilizing engine for RimM CTD in evolution. In contrast, the low sequence conservation of surface residues might result in functional distinctions between $M t b$ Rim $_{\mathrm{CTD}}$ and its orthologs.

\subsection{Structural Comparisons between MtbRim $M_{C T D}$ and Its Orthologs}

To investigate structural distinctions between $M t b$ RimM $M_{C T D}$ and its orthologs, we performed CTD-centered structural superpositions based on sequence alignments. Surprisingly, coordinate RMSD was no more than $1.992 \AA$ for the orthologs despite low sequence identity, showing a high degree of structural similarity among the CTDs of RimM (Figure S1). The CTD of TthRimM, the one with the largest RMSD to MtbRimM ${ }_{C T D}$, displayed notable structural distinctions in the $\beta 3-\beta 4$ loop. In $M t b$ Rim $M_{C T D}$, the shorter $\beta 3-\beta 4$ loop showed less flexibility and took on a turn-like conformation (Figure $3 \mathrm{~A}$ ). In TthRimM, residues R129-R133 in this loop form a short $3_{10}$-helix (Figure 3B). By contrast, the S19-complexed TthRimM showed a reduced turn-like loop instead of a helix (Figure 3C), 
possibly hampered by spatial hindrance (Q56 of TthS19 to E135 of TthRimM) or electrostatic repulsion (K32 of TthS19 to R131 of TthRimM). Given that the $\beta 3-\beta 4$ loop in TthRimM underwent significant conformational change once the protein binding S19, whether this loop in $M t b$ RimM $M_{\mathrm{CTD}}$ showed a similar pattern is worthy of examination.

A

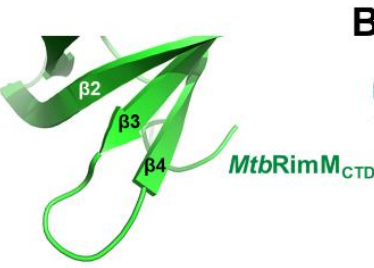

B

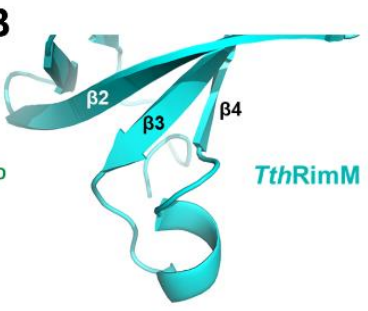

D

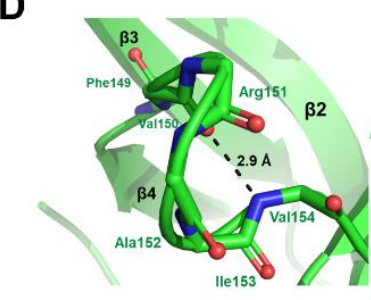

E

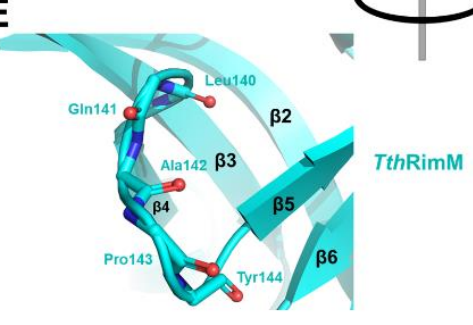

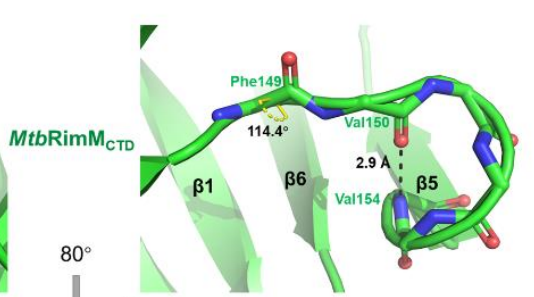
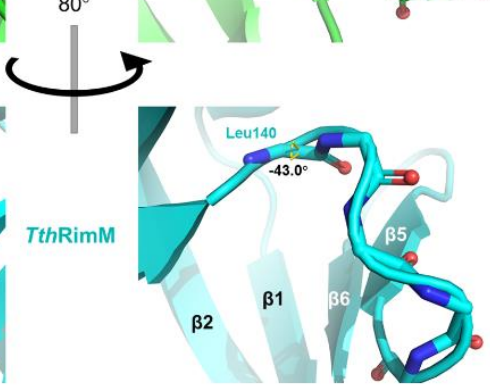

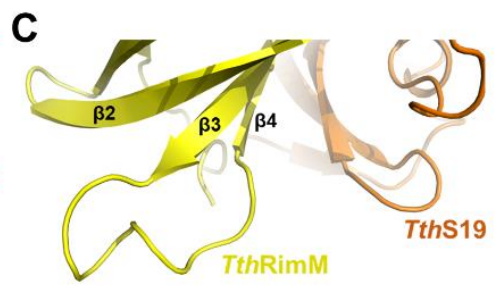

$\mathbf{F}$

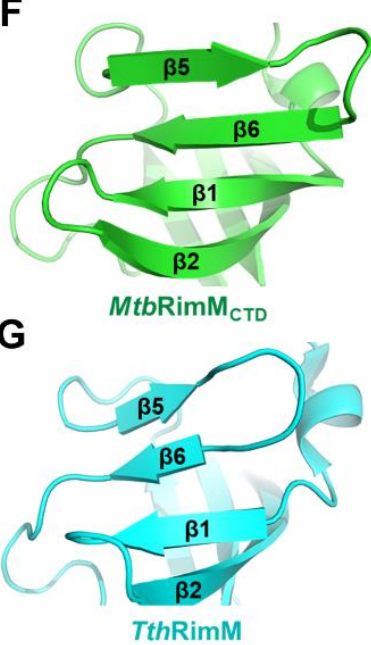

Figure 3. Structural comparison between $M t b R$ RimM $_{\mathrm{CTD}}$ and RimM from T. thermophilus HB8 (TthRimM) represented in cartoon. (A-C) $\beta 3-\beta 4$ loop of $M t b \operatorname{RimM} M_{\mathrm{CTD}}(\mathbf{A})$, free $T$ thRimM (B), S19-complexed TthRimM (C). (D,E) $\beta 4-\beta 5$ loop of $M t b \operatorname{RimM}_{\mathrm{CTD}}$ (D) and free TthRimM (E). The dihedral angle $\psi$ of F149 in (D) and L140 in (E) are identified. The length of the hydrogen bond (V154)N-H ... O(V150) is also depicted in (D), where backbone oxygen or nitrogen atoms are shown as red and blue sticks, respectively. Hydrogen atoms, if applicable, are hidden. (F,G) $\beta 5$ and $\beta 6$ strands of $M t b \operatorname{RimM} M_{\mathrm{CTD}}(\mathbf{F})$ and free $T \operatorname{th} \operatorname{RimM}(\mathbf{G})$.

On the other hand, the $\beta 4-\beta 5$ loop in $M t b$ RimM $_{\text {CTD }}$ consisted of a helix-like fold, characterized by the hydrogen bond between V150 and V154 (Figure 3D). This hydrogen bond also exists among the RimM orthologs with known structures, except for TthRimM. In fact, despite the backbone $\mathrm{N}-\mathrm{C}_{\alpha}$-C atoms of L140 in TthRimM adopted a similar orientation to the corresponding F149 in MtbRimM $\mathrm{CTD}_{\mathrm{CD}}$, their dihedral angles $\psi$ differed by $157.4^{\circ}$ (Figure 3D,E). This discrepancy, followed by conformational changes of other downstream residues, directly rendered the $\beta 4-\beta 5$ loop in TthRimM incapable of forming a dextro-fold and consequently the hydrogen bond. Additionally, taking the conservative V154, V157 and L159 in $\beta 5$, and V164 and I166 in $\beta 6$ of $M t b$ RimM $_{\mathrm{CTD}}$ as references, the shorter $\beta 4-\beta 5$ loop in TthRimM forced $\beta 5$ and even $\beta 6$ to contract toward the center of CTD (Figure $3 F, G$ ). It seemed that the $\beta 5$ and $\beta 6$ strands of $M t b \operatorname{RimM}_{\mathrm{CTD}}$ were more extended than TthRimM, supposedly contributing to the relative higher stability of the $\beta$-barrel.

\subsection{Backbone Relaxation Measurements of MtbRimM $M_{C T D}$}

To address dynamics features of $M t b$ RimM $_{\mathrm{CTD}}$, we performed NMR relaxation measurements of backbone amide groups to obtain longitudinal relaxation rates $\left(R_{1}\right)$, transverse relaxation rates $\left(\mathrm{R}_{2}\right)$, and $\left\{{ }^{1} \mathrm{H}\right\}-{ }^{15} \mathrm{~N}$ heteronuclear steady-state nuclear Overhauser effects (hNOE) (Figure 4A). A total of 68 backbone amide resonances were analyzed to characterize internal motions of $M t b \operatorname{RimM} M_{C T D}$. 


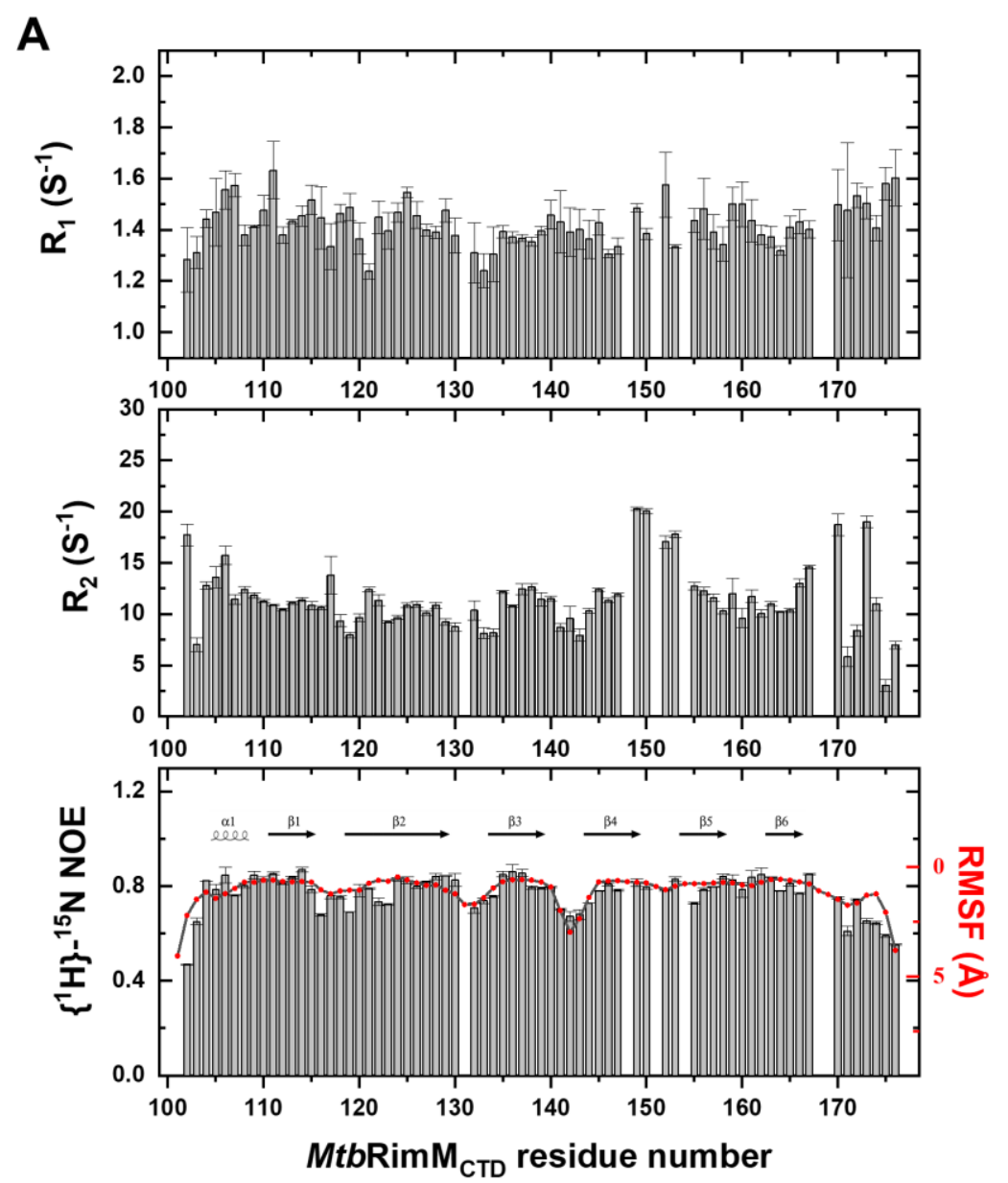

B

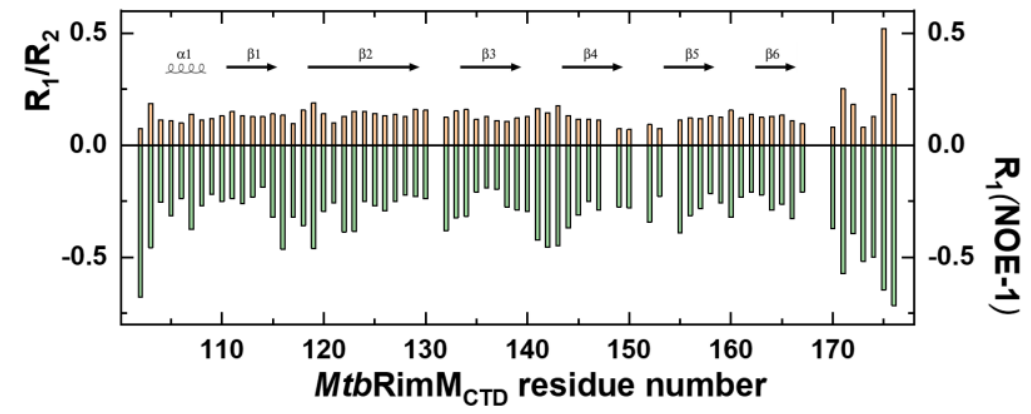

Figure 4. Nuclear magnetic resonance (NMR) relaxation measurements of backbone amide groups in $M t b$ RimM $M_{\mathrm{CTD}}$. (A) Plots of backbone amide dynamics parameters $\mathrm{R}_{1}$ (upper panel), $\mathrm{R}_{2}$ (middle panel), and $\left\{{ }^{1} \mathrm{H}\right\}-{ }^{15} \mathrm{~N}$ heteronuclear steady-state nuclear Overhauser effect (hNOE, lower panel) versus residue number. Root-mean-squared fluctuation (RMSF) per residue calculated from molecular dynamics (MD) simulation is plotted over the hNOE graph, as both parameters reveal fast motion features in line with the secondary structure elements shown above the plot. (B) Plot of the $R_{1} / R_{2}$ ratio and $R_{1}(\mathrm{NOE}-1)$ value versus residue number. Cross-relaxation rate $\sigma_{\mathrm{HN}}$ is characterized by $\mathrm{R}_{1}$ (NOE-1) for clearly comparing with the $\mathrm{R}_{1} / \mathrm{R}_{2}$ ratio. Secondary structure elements are shown above the column plot.

$\mathrm{R}_{1}, \mathrm{R}_{2}$, and $\mathrm{hNOE}$ are generally used to reflect residue-specific dynamics of the protein. The global average of hNOEs was 0.77 , indicating the compactness of the $\beta$-barrel. Overall, residues in the loops exhibited smaller $\mathrm{R}_{2}$ and hNOE values than those on the $\beta$-sheet, implicated in significant conformational flexibility. Plotting $R_{1} / R_{2}$ and cross-relaxation rate against sequence number provided a more intuitive understanding of backbone dynamics (Figure $4 \mathrm{~B}$ ), where the strand-interval loops showed faster dynamics than the $\beta$-strands. 
Exceptions were several residues near the $\beta 4$ - $\beta 5$ loop (residues $149-153$ ) and the C-terminal tail (D102, H170 and L173), as these residues displayed large $\mathrm{R}_{2}$ values disproportional to their due flexibilities. Since the apparent $R_{2}$ contains, if any, a conformational exchange term $R_{e x}$ [42], these loop residues were likely to be involved in internal motion on the $\mu$ s-ms timescale.

The $\beta$-sheet included most of the dynamically stable residues according to the relaxation data (Figure 4A,B). As expected, the residues forming the hydrophobic core benefited from the clustering of their non-polar side chains, and showed better rigidity than other residues. Only seven residues in the $\beta$-sheet (E119, G122, V123, E134, E144, and T155) which were hydrophilic or located on the protein surface had hNOE values lower than the global average, confirming our speculation.

Then, we evaluated the overall rotational correlation time $\left(\tau_{c}\right)$ of $M t b \operatorname{Rim} M_{C T D}$ to be $5.87 \pm 0.40$ ns based on the $R_{2} / R_{1}$ data of the residues situated in both the $\alpha 1$ helix and $\beta$-sheet [43]. Given the rough linear correlation between $\tau_{\mathrm{c}}$ and molecular weight $\left(\mathrm{M}_{\mathrm{w}}\right)$ on basis of empirical measurements [43], the molecular weight of $M t b R i m M_{C T D}$ was estimated to be $9.90 \mathrm{kDa}$. This estimated $\mathrm{M}_{\mathrm{w}}$ well conformed to the theoretical $\mathrm{M}_{\mathrm{w}}$ of recombinant $M t b \operatorname{RimM}_{\mathrm{CTD}}(9.28 \mathrm{kDa})$, implying that this protein existed in solution as a monomer.

\subsection{Model-Free Analysis for Backbone Dynamics of MtbRimM $M_{C T D}$}

To further comprehend dynamics features of $M t b \operatorname{Rim}_{\mathrm{CTD}}$, we calculated residuespecific dynamics parameters including the generalized order parameter $S^{2}$, the correlation time of internal motions $\tau_{e}$, and the conformational exchange rate $R_{e x}$, based on the measured $R_{1}, R_{2}$, and hNOE values by using the FAST-Modelfree program $[26,44,45]$. We adopted an axially-symmetric rotational diffusion tensor to initiate the iterations. The overall rotational correlation time $\tau_{\mathrm{c}}$ was fitted to be $6.19 \pm 0.03 \mathrm{~ns}$, which was in good agreement with our previous outcome based on the $R_{2} / R_{1}$ ratios, with errors taken into account. The critical diffusion tensor parameter $\mathrm{D}_{\text {ratio }}$ was determined to be 1.05 , implying that this protein could be dynamically described using a globular model. A detailed graph for these dynamics parameters is shown in Figure 5. As A131, R151, and three prolines were absent in the 2D NMR spectra for relaxation measurements, 65 out of 71 residues were successfully assigned to different motional models.

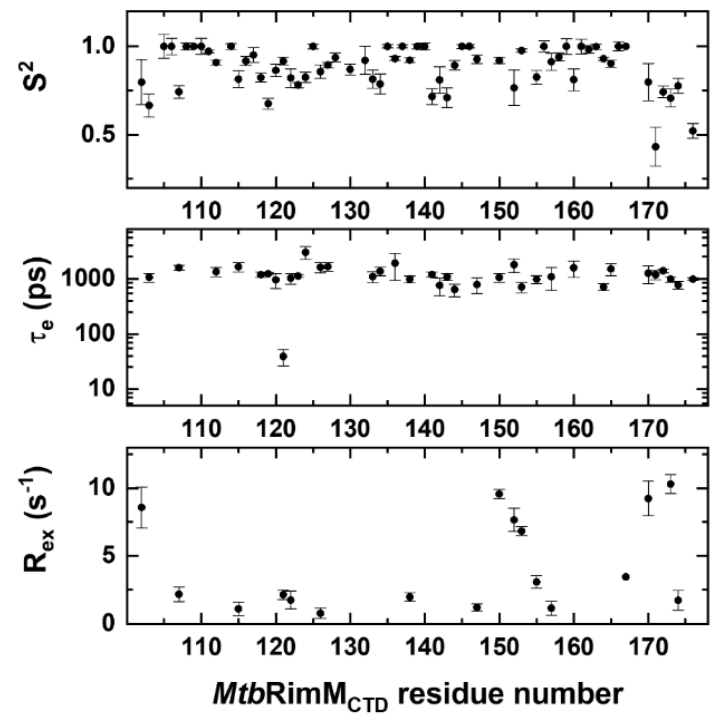

Figure 5. Dynamics parameters of $M t b R i m M_{C T D}$ obtained from Model-free analysis of NMR relaxation data. Residue-specific dynamics parameters $S^{2}$ (upper panel), $\tau_{\mathrm{e}}$ (middle panel), and $R_{\mathrm{ex}}$ (lower panel) are plotted per residue. Residues with either lower $\tau_{e}$ values or $R_{e x}$ values than their respective errors are not presented in the graphs. 
The average value of $S^{2}$ was 0.87 , indicating that $M t b R i m M_{C T D}$ adopted a rigid fold. Collectively, the $S^{2}$ values showed fluctuations consistent with the relaxation data. In particular, the residues in $\alpha 1$ helix except for Q107 exhibited the highest $S^{2}$ value of 1 , while Q107 displayed apparent flexibility $\left(S^{2}=0.743 \pm 0.035\right)$ (Figure 5). Furthermore, Q107 was the unique residue within $\alpha 1$ helix of which the motion pattern required both $\tau_{\mathrm{e}}$ and $R_{e x}$ values to describe, suggesting this residue underwent fast internal motion (ps-ns) and intermediate conformation exchange ( $\mu \mathrm{s}-\mathrm{ms}$ ). Moreover, 7 out of 11 residues in $\beta 2$ strand, the longest $\beta$-strand, displayed $S^{2}$ values lower than 0.9 and presented nanosecondtimescale internal motions. Note that each half of the long $\beta 2$ strand was stabilized by the shorter $\beta 1$ and $\beta 3$ strands via hydrogen bonding, respectively (Figure $2 \mathrm{~A}$ ).

In essence, $R_{\text {ex }}$ characterizes conformational exchange on the $\mu \mathrm{s}-\mathrm{ms}$ timescale if applicable and exists as a linear term of $R_{2}$ [42]. Reevaluation of $R_{2}$ by stripping it of possible $R_{\text {ex }}$ was thus feasible for the loop residues with excessive $R_{2}$ rates (D102, H170, and L173 in the terminal loops, and V150, A152, and I153 in the $\beta 4-\beta 5$ loop). These residues showed high $R_{e x}$ values up to $10.3 \mathrm{~s}^{-1}$, signifying that their intrinsic $R_{2}$ values were overestimated (Figure 5). Furthermore, the $R_{\mathrm{ex}}$ values in the fragment of V150-V157 exhibited a gradual downward trend, implying that the $\beta 4-\beta 5$ loop experienced overall conformational exchange, which was primarily stabilized by the downstream $\beta 5$ strand.

\subsection{Simulations of MtbRim $M_{C T D}$}

We further explored the structural stability of $M t b R i m M_{C T D}$ based on dynamics enlightenment by the NMR relaxation data. To this end, we performed a 120-ns molecular dynamics (MD) simulation starting with the determined solution structure. Indeed, the root-mean-squared fluctuation (RMSF) of backbone amide atoms $(\mathrm{N}-\mathrm{H})$ in residues well matched the experiment-derived hNOEs, especially for the inter-strand loops (Figure 4A). The average value of RMSF reached $1.08 \AA$, highlighting the rigidity of $M t b R i m M_{C T D}$ as a whole. Impressively, residue S142 in the $\beta 3-\beta 4$ loop showed a large RMSF second only to the two terminal residues D101 and E176, conforming to the low conservation of this loop in both sequence and structure.

The above-described model-free analysis revealed that the $\beta 4-\beta 5$ loop was subjugated to intermediate conformational exchange. During the MD simulation, we observed that the dihedral angles $\psi$ of F149 and $\varphi$ of V150 both had two different values (Figure 6A-C), while those for the residues 151-155 showed merely minor fluctuations (Figures S2 and S3). Notably, the $\psi$ distinction of F149 influenced the structure of the $\beta 4-\beta 5$ loop, as indicated by the local structural distinction between $M t b \operatorname{Rim}_{\mathrm{CTD}}$ and TthRimM CTD. To examine whether the helix-like fold of the $\beta 4-\beta 5$ loop could undergo loosening, we accessed the time evolution of the V154-V150 hydrogen bond. Intriguingly, the bond length and the N-H ... O bond angle remained practically stable throughout the MD simulation (Figure 6D-F), depicting the helix-like structure of the $\beta 4-\beta 5$ loop as a stable entity. Together, these restrictions described a model in which the conformational exchange of the $\beta 4-\beta 5$ loop was preferably integral than residue-wise (Figure 6G,H).

The 120-ns MD simulation also provided insight into stabilities of the secondary structure elements contained in MtbRimM $M_{\text {CTD }}$. As shown in Figure S4, the $\alpha$-helix and $\beta$-strands were generally stable during the simulation. Several inter-strand loops formed turns, further consolidating the rigidity of the protein structure. These MD assessments, combined with the determined structure and NMR relaxation data described above, were indicative of overall well-folded $M t b R$ RimM $_{\mathrm{CTD}}$ in solution, in contrast to the partly folded TthRimM CTD in solution [13]. 


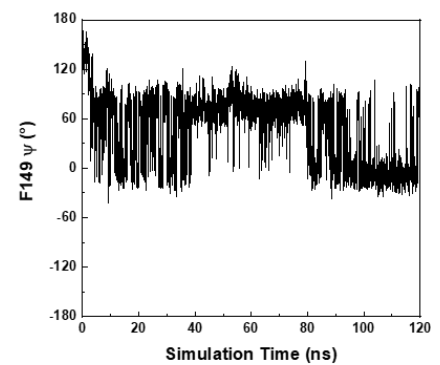

D

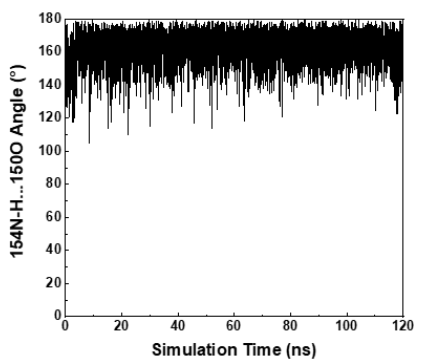

G

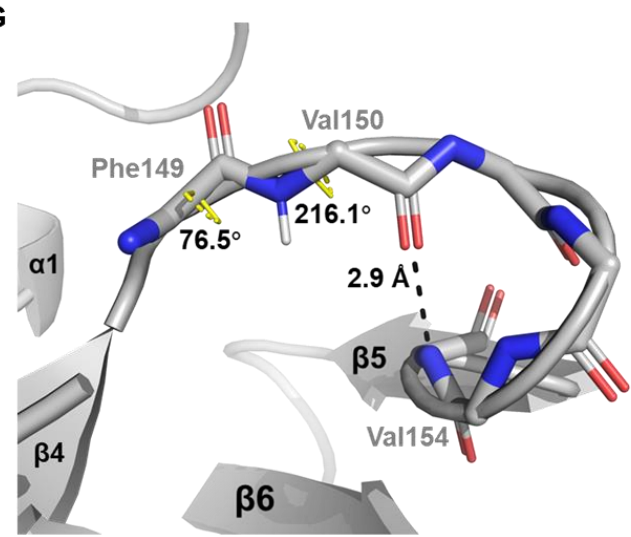

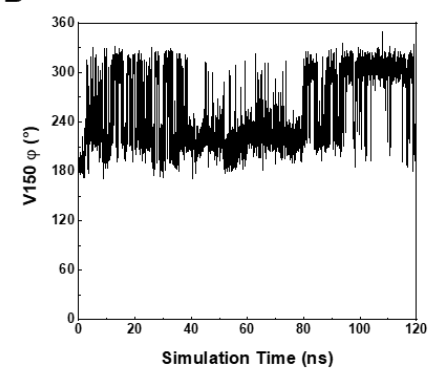

E

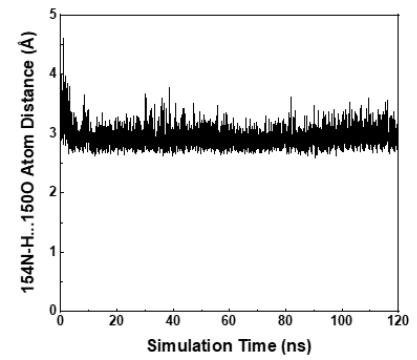

C

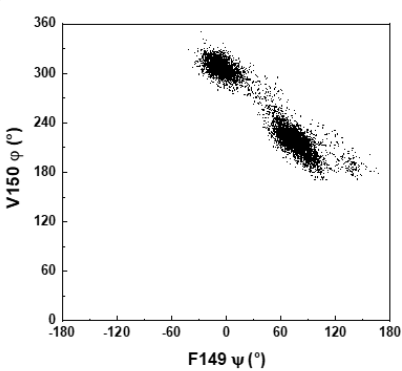

F

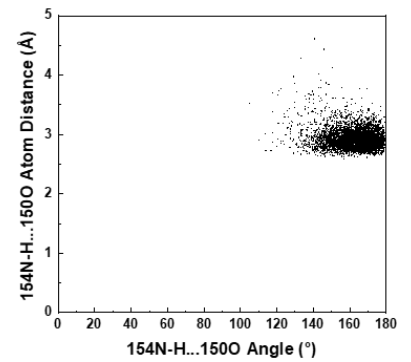

H

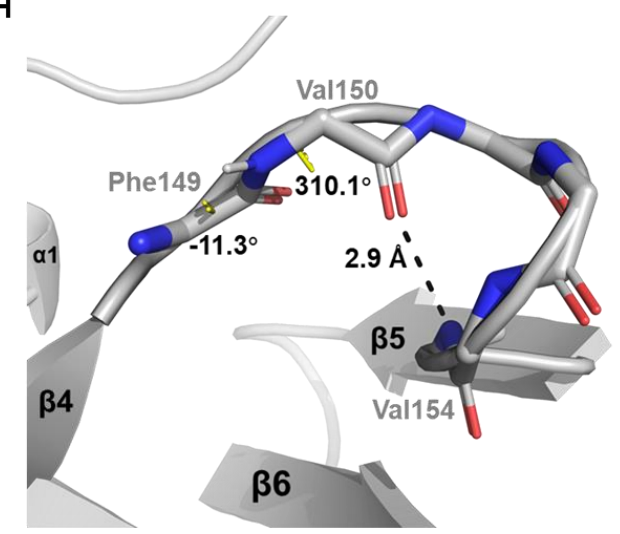

Figure 6. In silico dynamics features of $M t b \operatorname{RimM}_{\mathrm{CTD}}$ revealed by MD simulation. (A,B) Fluctuations of critical backbone dihedral angle F149 $\psi($ A) and V150 $\varphi$ (B) in the $\beta 4-\beta 5$ loop. F149 $\psi$ is scaled to $\left(-180^{\circ}, 180^{\circ}\right)$ and V150 $\varphi$ to $\left(0^{\circ}, 360^{\circ}\right)$ to avoid aliases. (C) 2D plot of V150 $\varphi$ vs. F149 $\psi$. The connection between F149 and V150 adopts two major orientations, as shown correspondingly in the plot. (D-F) Stability of the hydrogen bond (V154)N-H ... O(V150) indicated in Figure 3D. Both the hydrogen bond angle (D) and hydrogen bond length (E) remain almost constant in the MD simulation. The 2D plot of hydrogen bond length vs. hydrogen bond angle $(\mathbf{F})$ is also implicated in a stable hydrogen bond. $(\mathbf{G}, \mathbf{H})$ Structural snapshots for depicting the motion of the $\beta 4-\beta 5$ loop at two simulation time of $78.14 \mathrm{~ns}(\mathrm{G})$ and $106.10 \mathrm{~ns}(\mathbf{H})$. V150 $\varphi$-F149 $\psi$ exhibits two pairs of typical values, while the downstream helix-like fold undergoes motion as an undistorted entity. Hydrogen atoms are hided except for the V150 backbone amide ${ }^{1} \mathrm{H}$ atom in each frame.

\subsection{Affinity Assessment and Binding Sites Mapping of MtbRimM $M_{C T D}$ against S19}

Based on the determined structure of $M t b \operatorname{Rim} M_{\mathrm{CTD}}$, we exploited the interaction between $M t b$ RimM $M_{C T D}$ and S19. Thus, we obtained the recombinant S19 protein from $M t b$ (residues 1-93, termed MtbS19), and performed SPR assays to assess the affinity of $M t b \operatorname{Rim} M_{\mathrm{CTD}}$ for binding S19. The dissociation constant $\left(\mathrm{K}_{\mathrm{D}}\right)$ of the $M t b \mathrm{RimM}_{\mathrm{CTD}}-\mathrm{S} 19$ interaction was measured to be $2.16 \mu \mathrm{M}$ (Figure 7A), indicative of an intermediate-range interaction. Then, we conducted the equimolar NMR titration of S19 to MtbRimM $\mathrm{CTD}_{\text {. By }}$ comparing the ${ }^{1} \mathrm{H}_{-}{ }^{15} \mathrm{~N}$ HSQC spectra of $M t b$ RimM ${ }_{\mathrm{CTD}}$ with and without S19, we observed major peak changes (displacement, line broadening, and vanishment) related to backbone amide groups of $M t b \operatorname{Rim} M_{\mathrm{CTD}}$, which indicated an interaction of $\mathrm{mM}-\mu \mathrm{M}$ magnitude con- 
forming to the result of SPR assay (Figure 7B). Vanished resonances were associated with the following residues (102-109, 129-136, 145, 147-151, 153, 155, 157, 165, and 170-176), implicated in intermediate conformational exchanges once S19 binding (Figure 7A). We plotted backbone amide chemical shift perturbations (CSPs) of the remaining peaks, and marked the residues with significant CSPs (Figure 7C). Mapping the significantly changed residues to the 3D structure of $M t b$ RimM $M_{C T D}$ showed that the S19 binding sites encompassed nearly one-half of the $\beta$-barrel (Figure 7D).

A

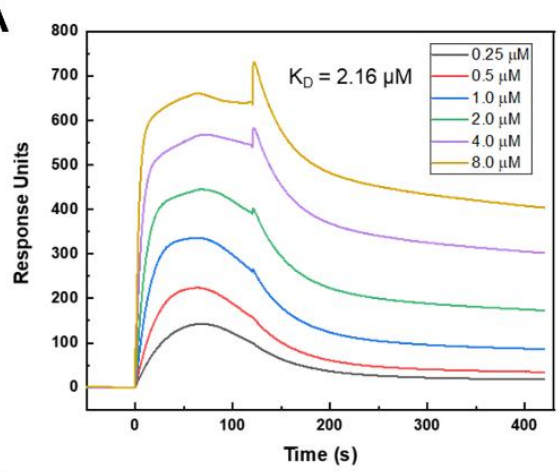

B

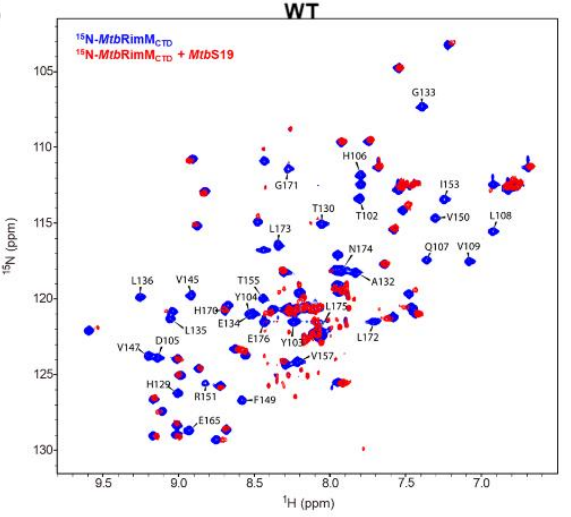

C

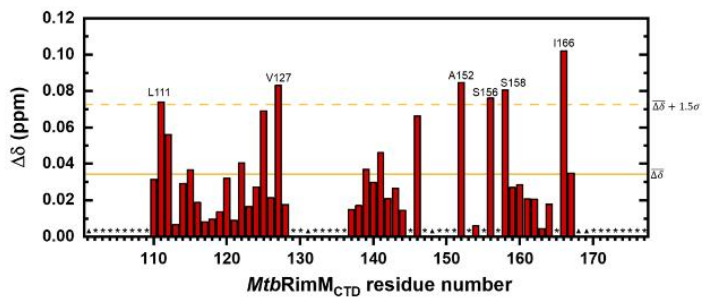

D

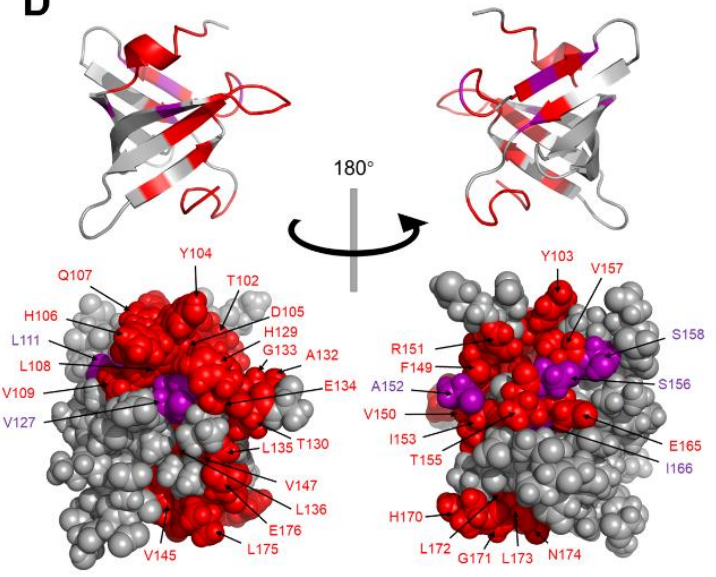

Figure 7. Interaction between $M t b \operatorname{RimM} M_{C T D}$ and $M t b S 19$. (A) Surface plasmon resonance (SPR) affinity assay of $M t b$ RimM $M_{C T D}$ binding S19 at serial concentrations. Blank control had been deducted from the serial data. (B) Overlapped ${ }^{1} \mathrm{H}_{-}{ }^{15} \mathrm{~N}$ heteronuclear singular quantum correlation (HSQC) spectra of ${ }^{15} \mathrm{~N}$-labeled $M t b$ RimM ${ }_{\mathrm{CTD}}$ alone (blue) and in presence of equimolar MtbS19 (red) for NMR titration assay. Peaks experiencing broadening-induced disappearance are indicated. (C) Plot of chemical shift perturbations (CSPs, $\Delta \delta$ ) of backbone amide groups. The mean value is indicated by a solid line, and the mean value plus 1.5 standard deviations by a dashed line. Asterisks indicate residues with disappear peaks at the titration destination, while triangles denote residues with invisible resonances before the titration, including D101, A131, and three prolines (residues 148, 168, and 169). (D) Mapping the binding surface to the 3D structure of MtbRimM $M_{C T D}$. Disappeared peaks are colored in red, and peaks with large CSPs (above the dashed line in $(\mathbf{C})$ ) in purple. Upper and lower panels are cartoon and sphere depictions of the structure, respectively.

\subsection{Molecular Docking of the MtbRimM $M_{C T D}-$ S19 Complex}

To establish a structural model of the $M t b \operatorname{RimM}_{\mathrm{CTD}}-\mathrm{S} 19$ complex, we carried out flexible protein-protein docking of S19 into MtbRimM $M_{C T D}$. Although the MtbS19 structure is available as a part of the 70S ribosome structure (PDB: 5V93) [46], residues 84-93 in the C-terminal tail of S19 are absent in the ribosome structure. Given that the corresponding fragment in TthS19 directly contacts TthRimM as displayed in the 3D structure of the TthRimM-S19 complex (PDB: 3A1P), using the structural component of MtbS19 contained in the 5V93 structure as a template might harm the confidence level of the molecular docking. Considering that S19-based structural superposition between the 5V93 and 3A1P structures gave a small backbone RMSD of $1.08 \AA$ (Figure S5), we used the structural 
component of TthS19 contained in the 3A1P structure as a template to build the structural model of $M t b S 19$. With this structural model and the solution structure of $M t b$ RimM $\mathrm{CTD}_{\mathrm{D}}$, we established the structural model of the $M t b$ RimM $_{\mathrm{CTD}}-\mathrm{S} 19$ complex by molecular docking.

Out of 30,000 structural models generated for the $M t b R_{\text {Rim }} \mathrm{CTD}_{\mathrm{C}}-\mathrm{S} 19$ complex, the one with an optimized interface energy score of -61.563 (hereafter referred to as the docking model) was used for the following assessment (Figure 8A). An inspection into the surface electrostatic potentials of $M t b$ Rim $M_{C T D}$ and S19 in the docking model indicated extensive electrostatic potential distributions in the binding interfaces on both proteins (Figure 8B). The negatively charged belt in the $M t b$ Rim $M_{C T D}$ surface tightly stuck to the positively charged ring of S19, indicating that electrostatic interaction played a predominant role in $M t b$ RimM $M_{\text {CTD }}$ binding S19. Several residues in MtbRimM $M_{\text {CTD }}$ (D105, E126, H129, A132, D143, E144, and R151) were involved in direct interactions with S19 (Figure S6), most being charged residues. Hydrophobic interactions also existed between the exposed non-polar parts of both proteins. These results conformed to the binding sites mapped by NMR titration assay described above, which underlined the crucial role of surface electrostatic interactions in minimizing the interface energy of the $M t b \operatorname{RimM}_{\mathrm{CTD}}-\mathrm{S} 19$ complex.
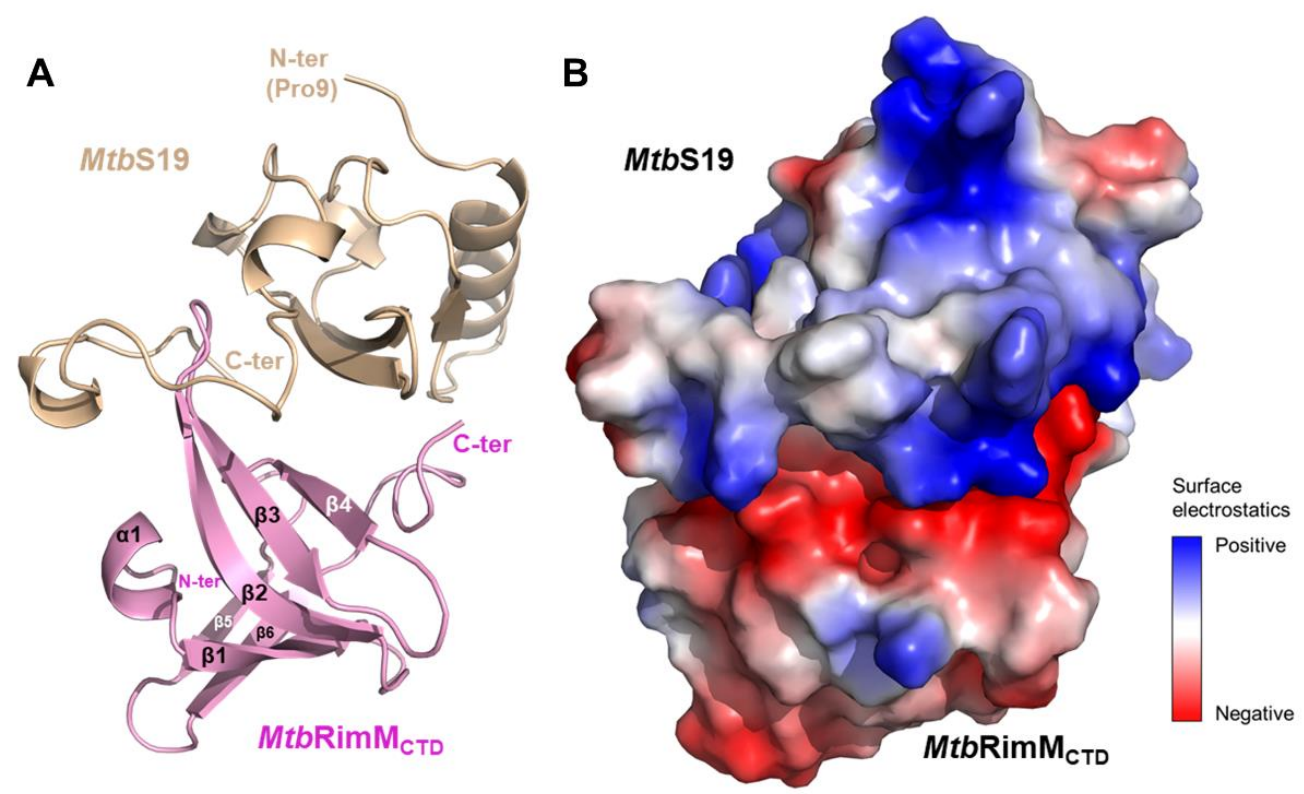

Figure 8. Molecular docking model of the MtbRimM $\mathrm{CTD}^{-S 19}$ complex. (A) Cartoon depiction of the docking model. The structure of MtbS19 (residues 9-93, light brown) was modeled using the crystal structure of TthRimM-complexed TthS19 (PDB: 3A1P) as the template. (B) Surface electrostatic potentials of the docking model. The binding interface is mainly composed of charged residues. Positively charged residues are primarily from the long loop located near the C-terminus of MtbS19, and negatively charged residues mostly from $M t b \operatorname{Rim} M_{\mathrm{CTD}}$.

Notably, the imidazole group of $\mathrm{H} 129$ at the end of the $\beta 2$ strand in $M t b$ RimM $M_{\mathrm{CTD}}$ formed hydrogen bonds with both the backbone oxygen of $\mathrm{H} 83$ and side-chain amide of R88

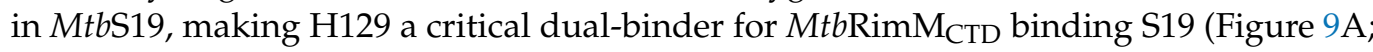
Figure S6). Meanwhile, MtbRimM $\mathrm{CTD}_{\mathrm{C}} \mathrm{D} 105$ in $\alpha 1$ helix formed a hydrogen bond with MtbS19 K85 in the short helix. Structural superposition displayed that the C-terminal tail of MtbS19 in the docking model of the MtbRimM-S19 complex underwent a displacement relative to the TthRimM-S19 complex. On the other hand, TthRimM D114 (corresponding to $M t b \operatorname{Rim} M_{\mathrm{CTD}} \mathrm{H129}$ ) also served as a dual-binder, forming two salt bridges with the side chains of TthS19 K88 and K91 (Figure 9B). Furthermore, the carbonyl group of TthRimM Y91 (corresponding to $M t b R i m M_{C T D}$ D105) formed a hydrogen bond with the backbone amide group of TthS19 K88. Sequence alignment showed that neither D105 nor H129 in $M t b \operatorname{RimM}$ were conserved in other RimM orthologs (Figure 1B). It seemed that $M t b \operatorname{RimM}$ 
D105 and H129 might contribute to the unique pattern of MtbRimM $M_{\mathrm{CTD}}$ binding S19, adopting different hydrogen binding modes to bind S19 from TthRimM Y91 and D114.

A

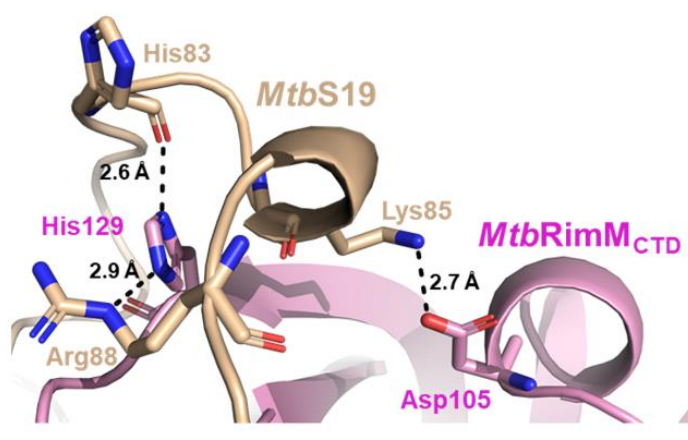

C

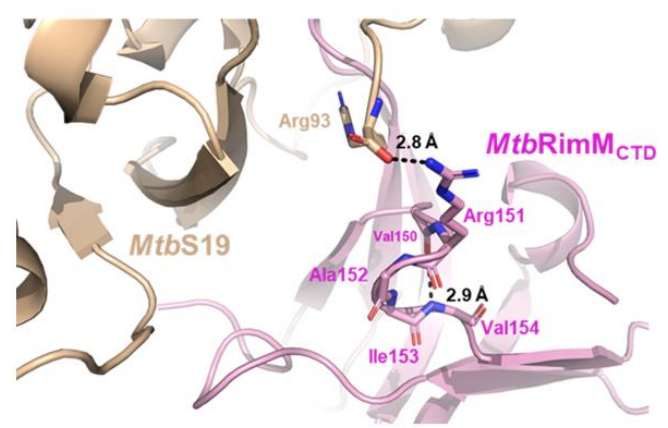

B

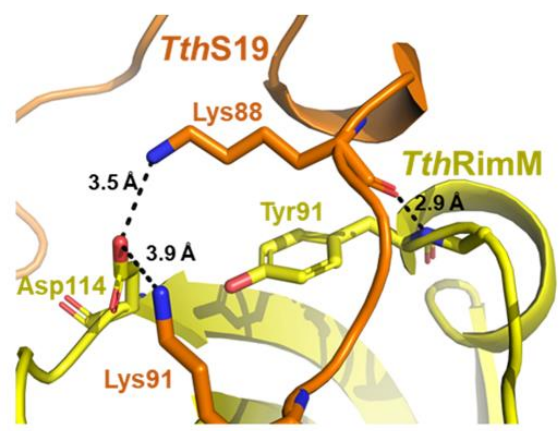

D

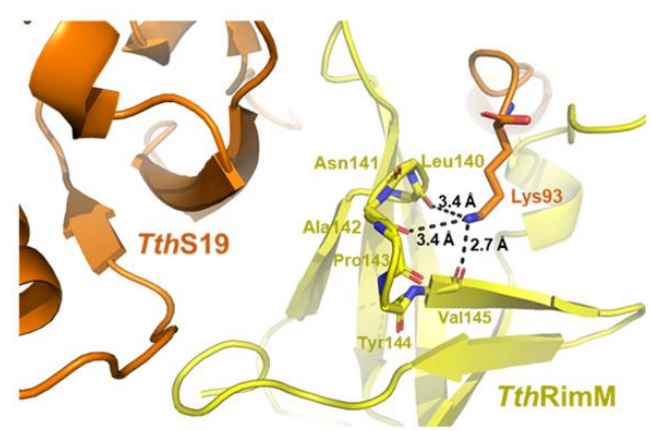

Figure 9. Comparison between the docking model of $M t b \operatorname{RimM}_{\mathrm{CTD}}-\mathrm{S} 19$ complex and the crystal structure of TthRimM-S19 complex. (A,B) Illustration of the stabilized short helix located in the long C-terminal loop of S19 and nearby residues within the docking model of $M t b$ RimM $_{\mathrm{CTD}^{-}}-\mathrm{S} 19$ (A) or the crystal structure of TthRimM-S19 (PDB: 3A1P) (B). Atom distances are shown beside the corresponding black dashed lines. (C,D) S19 C-terminal residue Arg93 involved in the interaction

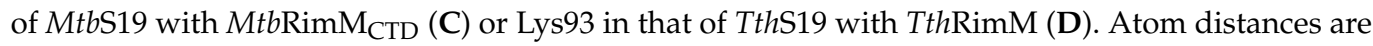
shown beside the corresponding black dashed lines.

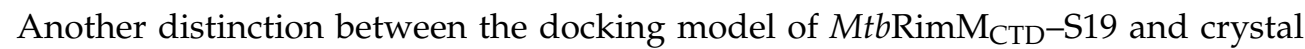
structure of TthRimM-S19 lay in the $\beta 4-\beta 5$ loop. The oxygen atoms of backbone carbonyls of TthRimM L140, A142, and V145 in the $\beta 4-\beta 5$ loop encircled the side chain amide atoms of TthS19 K93, upholding the flexible C-terminus (Figure 9D). However, this interaction pattern did not work for $M t b \operatorname{RimM}_{\mathrm{CTD}}$, potentially owing to the structural distinction in the $\beta 4-\beta 5$ loop between $M t b$ RimM $M_{C T D}$ and TthRimM. The docking model of MtbRimM-S19 displayed an alternative binding pattern in which a hydrogen bond was formed between the guanidine group of $M t b R i m M ~ R 151$ and carboxyl group of $M t b S 19$ R93 (Figure 9C; Figure S6). The structural distinction of the $\beta 4-\beta 5$ loop, together with the critical charge-reversive substitution at residue 129 (positively charged MtbRimM $M_{C T D}$ H129 vs. negatively charged TthRimM D104), might contribute the change in the interaction pattern of the C-terminal tail in MtbS19. In summary, the docking model of MtbRimM CTD $-\mathrm{S} 19$ was distinguished from the crystal structure of TthRimM-S19 primarily due to structure rearrangements between the two orthologs which were induced by pivotal residue replacements.

\subsection{Affinity Assessments for MtbRimM $M_{C T D}$ Mutants Binding $S 19$}

To verify the reliability of the docking model of $M t b R i m M_{C T D}-S 19$, we obtained sitedirected mutants of $M t b \operatorname{RimM}_{\mathrm{CTD}}$ at key loci and then measured their affinities for binding

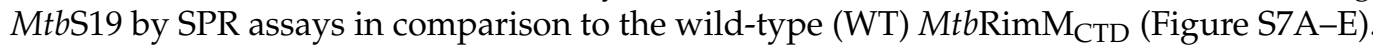
Mutation candidates included the nonconserved D105 and H129 considering their potential 
key roles in the unique interaction pattern of $M t b R i m M_{C T D}$ with S19, and the conservative E126, E134, and E144 joining in electrostatic interactions with S19. Since they functioned primarily with their charged side chains according to the docking model of $M t b R i m M_{\mathrm{CTD}^{-}}$ S19, these five residues were subjugated to alanine substitutions accordingly. As a result, all these mutants displayed 0.8-4.9 times increases in their $\mathrm{K}_{\mathrm{D}}$ values compared to $\mathrm{WT}$, signifying significantly decreased affinities (Figure 10). In particular, H129A exhibited the most considerable change of affinity among the mutants as indicated by the $\mathrm{K}_{\mathrm{D}}$ values $(12.68 \mu \mathrm{M}$ for H129A vs. $2.16 \mu \mathrm{M}$ for WT), agreeing with the dual-binder role of $M t b$ RimM H129 in the docking model, despite this nonconserved residue with diverse charges among the orthologs. The second-largest decrease in affinity was observed for MtbRimM D105A binding S19 $\left(\mathrm{K}_{\mathrm{D}}=7.06 \mu \mathrm{M}\right)$, followed by E144A and E126A. As a highly conservative component of the positively charged belt on the MtbRimM $M_{C T D}$ surface but not present on the S19 binding interface of the docking model, the E134A mutant showed the smallest change in $\mathrm{K}_{\mathrm{D}}$, as expected. These results indicated that the two unique residues D105 and H129 played vital roles in the interaction of MtbRimM ${ }_{\mathrm{CTD}}$ with S19, consolidating the creditability of the docking model of $M t b$ RimM $_{\mathrm{CTD}}-\mathrm{S} 19$.

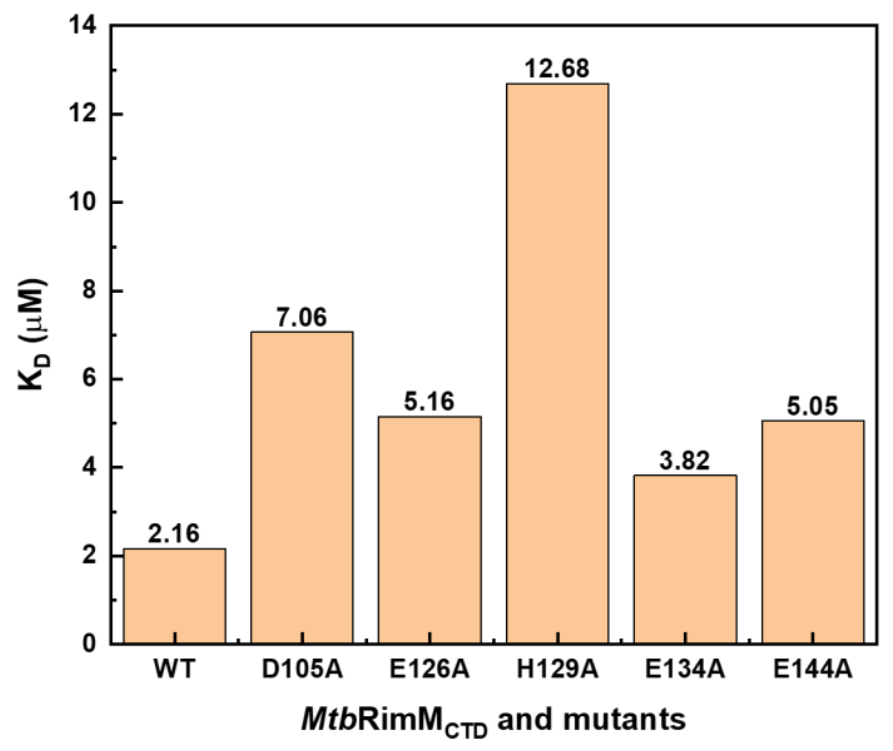

Figure 10. Dissociation constants $\left(\mathrm{K}_{\mathrm{D}}\right)$ of wild-type $(\mathrm{WT}) \mathrm{M} t b \operatorname{RimM}_{\mathrm{CTD}}$ and its mutants for binding MtbS19 determined by SPR affinity assays.

\section{Discussion}

TB is threatening the world healthcare with its MDR variants. Novel drug targets are urgently needed for effective prevention and treatment of MDR-TB. As is known, ribosomes are the organelle responsible for controlling protein biosynthesis. Critical protein components in bacterial ribosomes could be explored as potential drug targets [47]. As one of the regulation factors vital for $30 \mathrm{~S}$ ribosomal subunit assembly in vivo with S19-binding activities in vitro, RimM could be a potential target for the development of novel drugs against MDR-TB [5,8]. However, the 3D structure of MtbRimM has not yet been determined. Considering that the CTD is primarily responsible for the interaction of $M t b$ RimM with S19, we herein clarified the structural basis of $M t b$ RimM $M_{\text {CTD }}$ binding S19 by applying several biophysical techniques. Based on the mapped S19 binding sites on $M t b$ RimM $_{\mathrm{CTD}}$, we established the docking model of the $\mathrm{M} t b$ RimM $_{\mathrm{CTD}}-\mathrm{S} 19$ complex, and identified critical residues significantly contributing to the unique pattern of $M t b \operatorname{RimM}_{\mathrm{CTD}}$ binding S19. 


\subsection{MtbRimM CTD Is Structurally Independent of NTD and Primarily Responsible for Binding S19}

Prior to structure determination, we firstly confirmed structural independence between the CTD and NTD of MtbRimM. A previous study declared the structural independence of TthRimM CTD on NTD by comparing the NMR structure ensemble and protein dynamics of a standalone NTD truncation with the NTD part of the full-length ThtRimM [13]. To examine the structural independence, we separately recorded ${ }^{1} \mathrm{H}^{-15} \mathrm{~N}$

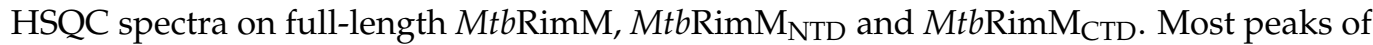
both $M t b$ RimM $\mathrm{NTD}_{\mathrm{NT}}$ and $M t b$ RimM $_{\mathrm{CTD}}$ overlapped well to those belonging to the full-length MtbRimM, implying that the domain truncation almost did not affect the structure of either CTD or NTD (Figure S8). Peaks of the N-terminal helix (residues 102-108) and inter- $\beta$ -

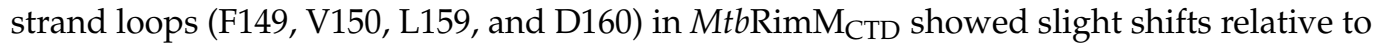
full-length $M t b$ RimM. Furthermore, the NMR titration of $M t b R_{\text {RimM }}$ NTD into $M t b$ RimM $_{\text {CTD }}$ and its reverse counterpart did not display observable peak changes (Figure S9). We thereby proved the structural independence between the CTD and NTD of MtbRimM. Ad-

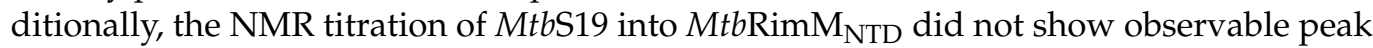
changes (Figure S10), implying that $\mathrm{S} 19$ basically did not bind to $M t b$ RimM $\mathrm{NTD}_{\mathrm{ND}}$. Together, these results allow us to exploit the structural basis of $M t b \operatorname{RimM}_{\mathrm{CTD}}$ binding S19 without NTD interference.

\subsection{Unique Features of $\beta 3-\beta 4$ and $\beta 4-\beta 5$ Loops Characterize a Well-Folded MtbRim $M_{C T D}$}

The solution structure of $M t b$ RimM $_{\mathrm{CTD}}$ is comprised of a six-strand $\beta$-barrel and a short $\alpha$-helix near the $\mathrm{N}$-terminus, characterized by a hydrophobic core and a predominantly negatively charged surface. The tightly packed core consists of conservative non-polar residues mostly located on the six $\beta$-strands. The overall rigidity of $M t b R i m M_{C T D}$ is reflected by the large all-residue mean $\mathrm{S}^{2}$ value of 0.87 (the generalized order parameter), and the small RMSF of $1.08 \AA$ in the MD simulation. Different from well-folded $M t b$ RimM $_{\mathrm{CTD}}$, TthRimM CTD is only partly folded in solution, as residues 125-143 showed few medium- or long-range NOEs [13]. However, crystal structures of both free TthRimM (PDB: 2DYI) and S19-complexed full-length TthRimM (PDB: 3A1P) display a CTD of $\beta$ barrel fold resembling $M t b$ RimM ${ }_{C T D}$, suggesting that the unfolded part of TthRimM CTD in solution was not stabilized until trapped in a local energy minimum during crystallization. In fact, all five crystal structures of RimM orthologs available in PDB exhibit general structural similarity to $M t b$ RimM $M_{C T D}$ despite low sequence identity. Interestingly, the fragment in MtbRimM ${ }_{C T D}$ corresponding to residues 125-143 in TthRimM starts from the end of $\beta 3$ and ends at the middle of $\beta 5$, covering the two major conformational discrepancies between the two orthologs in the $\beta 3-\beta 4$ loop and $\beta 4-\beta 5$ loop. Moreover, the highly flexible $\beta 3-\beta 4$ loop of $T$ thRimM CTD showed $\mathrm{R}_{1}$ values up to $4 \mathrm{~s}^{-1}$ and low hNOE values around 0.5 [13], in sharp contrast to the results obtained from the NMR relaxation measurement of $M t b R i m M_{\text {CTD }}$. Considering these results and the unique structural features of these two loops described above, the far shorter $\beta 3-\beta 4$ loop and the $\beta 4-\beta 5$ loop stabilized by a hydrogen bond (V154-V150) might facilitate the stable folding of $M t b$ RimM $M_{C T D}$.

\subsection{Both $\beta 4-\beta 5$ Loop and Nonconserved Key Residues Contribute to the Unique Pattern of MtbRimM $M_{C T D}$ Binding $S 19$}

The docking model of $\mathrm{M} t b \mathrm{RimM}_{\mathrm{CTD}}-\mathrm{S} 19$ shows an interface containing surface charged residues, where the positively charged C-terminal tail of MtbS19 binds to the negatively charged ring of $M t b \operatorname{RimM} M_{C T D}$ (Figure $8 \mathrm{~B}$ ). The docking model displays a binding pattern different from that identified from the crystal structure of TthRimM-S19 (PDB: 3A1P). The $\beta 4-\beta 5$ loop exhibits a smaller possibility to accommodate the C-terminus of $M t b S 19$ via multiple hydrogen bonds, forcing the terminal arginine to relocate to a different side of the 3A1P structure. Notably, conformational exchanges in this loop, which was intrinsically caused by the dihedral angle fluctuations of F149-V150, were revealed by NMR relaxation analysis and also confirmed by MD simulation (Figures 5 and $6 \mathrm{~A}-\mathrm{C}$ ). 
Comparatively, the experiment-based difference of the corresponding dihedral angle between MtbRimM CTD $_{149} \psi$ and TthRimM L140 $\psi$ was $157.4^{\circ}$, while the simulation-based fluctuation between the two dihedral angles individually related to two major orientations of $M t b \operatorname{RimM} \mathrm{CTD}_{\mathrm{CT}} \mathrm{F} 149 \psi$ or V150 $\varphi$ was nearly $90^{\circ}$ (Figure $6 \mathrm{~A}, \mathrm{~B}$ ). The orientation distinction, together with the stable hydrogen bond of V154-V150 (Figure 3D), might provide a mechanistic understanding of the unique role of the helix-like $\beta 4-\beta 5$ loop in $M t b R_{\text {RimM }} \mathrm{CTD}_{\mathrm{CT}}$ binding S19, which is distinct from that in TthRimM binding S19. Additionally, two non-

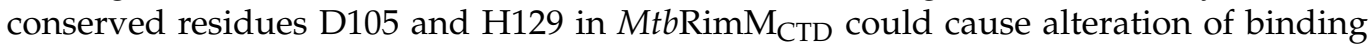
destination on the C-terminal tail of S19 (Figure 8A,B). Although MtbRimM $\mathrm{CTD}_{\mathrm{C}} \mathrm{H} 129$ displays reverse charge relative to its counterparts in RimM orthologs (e.g., D114 in TthRimM), its dual-binder function works well via the hydrogen bond rather than the salt bridge adopted by TthRimM D114, as evidenced by the largest affinity decrease of the H129A mutant observed by SPR assays. Hence, the docking model reveals a unique pattern of $M t b$ RimM $_{\mathrm{CTD}}$ binding S19.

Notably, some residues in MtbRimM $\mathrm{CTD}_{\mathrm{CT}}$ that displayed peak broadening in the NMR titration experiment seemed not to directly contact MtbS19 in the docking model of the $M t b R i m M_{\mathrm{CTD}^{-}}-\mathrm{S} 19$ complex. One of the potential reasons is that not only $\mathrm{M} t b \mathrm{RimM}_{\mathrm{CTD}^{-}}$ S19 interaction directly cause significant line broadening in residues on the S19-binding surface of $M t b R_{\text {RimM }} \mathrm{CTD}_{\mathrm{D}}$, but also conformational changes induced by either S19 binding or non-specific interactions could cause observable line broadening in several residues around the S19-binding surface. Expectedly, single-site mutation experiments on a case-by-case basis can help distinguishing the non-specific interactions from the specific interactions. Furthermore, a previous work provides comprehensive methods for identifying nonspecific interactions in the protein-ligand complex, especially the ligand-induced modulation [48].

\subsection{Partial Affinity Decrease of MtbRimM $M_{C T D}-S 19$ by Single-Site Mutation Calls for Efficient Binding Inhibitors}

While we have characterized the structural and interaction-related properties of $M t b$ RimM ${ }_{C T D}$, some intriguing facts might hamper efforts of $M t b$ RimM-based drug design. As described above, the single-site mutations of five residues (D105A, E126A, H129A, E134A, and E144A), no matter they were conservative residues or not, observably reduced but did not neutralize the affinity of $M t b$ RimM $_{\mathrm{CTD}}$ binding S19. This result suggests that single-site mutation is insufficient for full inhibition of the $M t b$ RimM $_{C T D}-S 19$ interaction. The logic behind this observation might lie in fault tolerance of RimM introduced during species evolution. Interestingly, even though RimM CTDs exhibit low conservation except for the residues forming the hydrophobic core, S19 orthologs among several bacterial species are highly conservative (Figure S11). In comparison, the human 40S ribosomal protein S15 shares a lower sequence identity with MtbS19 despite both proteins belong to the S19 protein family. Thus, relative low side effects on human hosts could be expected for potential drugs designed to specifically target Mycobacterium tuberculosis and significantly breaking the MtbRimM-S19 interaction. Additionally, the design of new anti-TB drugs eliminating the $M t b \operatorname{RimM}_{\mathrm{CTD}}-\mathrm{S} 19$ interaction should focus on aiming key residues on the S19-binding pockets in $M t b$ RimM $_{\mathrm{CTD}}$, or alternatively, on targeting the more conservative MtbS19.

Furthermore, we predicted the preliminary druggability of the $M t b \operatorname{RimM}_{\mathrm{CTD}}-\mathrm{S} 19 \mathrm{com}$ plex by using the PockDrug webserver [49] based on the docking model of $M t b$ RimM $_{\mathrm{CTD}^{-}}$ S19. The prediction identified two potential pockets, showing moderate druggability probabilities for both pockets (Figure S12, Table S1). Notably, pocket 1 involves MtbRimM $M_{\text {CTD }}$

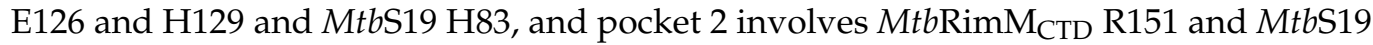
R93. These residues highly cover with the key residues on the interaction interface between $M t b$ RimM $_{\mathrm{CTD}}$ and S19, implicated in the pharmacal significance of $M t b$ RimM as a potential anti-TB drug target.

Expectedly, lead compounds capable of profoundly interfering with the hydrogen bonds and/or electrostatic interactions might inhibit the MtbRimM-S19 interaction and break this complex. Moreover, the convex hull volumes of both predicted drug pockets are 
in the order of $100 \AA^{3}$ (Table S1), indicative of the accommodation ability of small ligands. Taken together, these considerations imply that hydrophilic, charged, and slim molecules with capacities of blocking both drug pockets could be explored to be potential drugs targeting the MtbRimM-S19 interaction.

As the heteronuclear 3D NMR spectra recorded on full-length $M t b$ RimM exhibited severe peak overlap or peak broaden even disappearance, it is a difficult task to complete resonance assignments and structural determination for the full-length protein in solution. Nevertheless, we have demonstrated the structural independence between the NTD and CTD of MtbRimM. The current study on the standalone $M t b \operatorname{RimM}_{\mathrm{CTD}}$ will facilitate the future study on solution structure, dynamics and intermolecular interaction of full-length MtbRimM. Our results provide new insights into the molecular mechanisms of the RimM function regarding the assembly of S19 into the ribosome. Moreover, the structural basis of $M t b$ RimM ${ }_{\mathrm{CTD}}$ binding S19 revealed in this study may be beneficial to the development of novel drugs against MDR-TB.

Supplementary Materials: The following are available online at https://www.mdpi.com/article/ 10.3390/biom11040597/s1, Figure S1: Structural superpositions between MtbRimM CTD $_{\text {and RimM }}$ orthologs with known structures, Figure S2: Time evolutions of the backbone dihedral angle $\varphi$ of residues 151-155 in MtbRimM ${ }_{\mathrm{CTD}}$, Figure S3: Time evolutions of the backbone dihedral angle $\psi$

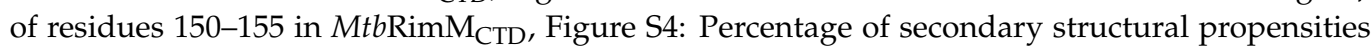
per residue predicted from the MD simulation, Figure S5: Structural superposition between the TthRimM-complexed TthS19 (PDB: 3A1P) and MtbS19 incorporated into the capreomycin-bound $70 \mathrm{~S}$ ribosome from M. tuberculosis (PDB: 5V93), Figure S6: Illustration of intermolecular interactions identified from the docking model of the $M t b R$ RimM $_{\mathrm{CTD}}-\mathrm{S} 19$ complex, Figure S7: SPR affinity assays of $M t b R i m M_{C T D}$ mutants binding $M t b S 19$ at serial concentrations, Figure S8: Overlapped ${ }^{1} \mathrm{H}^{-15} \mathrm{~N}$

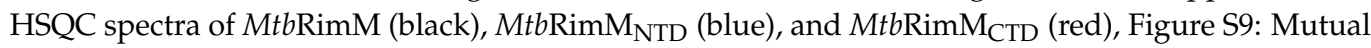
NMR titration assays between $M t b R$ RimM $_{\mathrm{CTD}}$ and $M t b$ RimM $_{\mathrm{NTD}}$, Figure S10: NMR titration assay of $M t b R i m M_{N T D}$ binding S19, Figure S11: Sequence alignments among MtbS19 and S19 orthologs corresponding to those for RimM CTDs, Figure S12: Prediction of potential drug pockets in the $M t b$ RimM $_{\mathrm{CTD}^{-}}-\mathrm{S} 19$ docking model via PockDrug server, Table S1: Parameters of predicted drug pockets in the MtbRimM $\mathrm{CTD}_{\mathrm{C}}-\mathrm{S} 19$ docking model via PockDrug server.

Author Contributions: Conceptualization, H.Z., Q.Z., C.G., H.W., and D.L.; methodology, H.Z., Q.Z., C.G., L.F., H.W., and D.L.; validation, H.Z., Q.Z., and D.L.; formal analysis, H.Z., Q.Z., C.G., L.F., and D.L.; investigation, H.Z., Q.Z., and X.L.; resources, D.L.; data curation, H.Z.; writing-original draft preparation, H.Z. and Q.Z.; writing - review and editing, H.Z., C.G., and D.L.; visualization, H.Z. and Q.Z.; supervision, D.L.; project administration, D.L.; funding acquisition, D.L. All authors have read and agreed to the published version of the manuscript.

Funding: This research was funded by the National Key Research and Development Project of China under grant No. 2016YFA0500600 and the Foundation for Innovative Research Groups of the National Natural Science Foundation of China under grant No. 21521004.

Institutional Review Board Statement: Not applicable.

Informed Consent Statement: Not applicable.

Data Availability Statement: The atomic coordinate file of $M t b R i m M_{\text {CTD }}$ presented in this study is openly available in the Protein Data Bank Japan (https://pdbj.org/) at https://doi.org/10.2210/ pdb7CQ1/pdb, reference number 7CQ1.

Acknowledgments: The authors are grateful to Junjie Chen of school of Pharmaceutical Sciences, $\mathrm{Xi}$ amen University for troubleshooting SPR assays. We also thank Hongwei Yao of Soochow University for the help with the NMR experiments.

Conflicts of Interest: The authors declare no conflict of interest. 


\section{References}

1. World Health Organization. Global Tuberculosis Report 2020; World Health Organization: Geneva, Switzerland, 2020.

2. Hicks, N.D.; Yang, J.; Zhang, X.; Zhao, B.; Grad, Y.H.; Liu, L.; Ou, X.; Chang, Z.; Xia, H.; Zhou, Y.; et al. Clinically prevalent mutations in Mycobacterium tuberculosis alter propionate metabolism and mediate multidrug tolerance. Nat. Microbiol. 2018, 3, 1032-1042. [CrossRef] [PubMed]

3. Goossens, S.N.; Sampson, S.L.; Van Rie, A. Mechanisms of Drug-Induced Tolerance in Mycobacterium tuberculosis. Clin. Microbiol. Rev. 2020, 34, e00141-e20. [CrossRef] [PubMed]

4. Guo, Q.; Goto, S.; Chen, Y.; Feng, B.; Xu, Y.; Muto, A.; Himeno, H.; Deng, H.; Lei, J.; Gao, N. Dissecting the in vivo assembly of the $30 \mathrm{~S}$ ribosomal subunit reveals the role of RimM and general features of the assembly process. Nucleic Acids Res. 2013, 41, 2609-2620. [CrossRef] [PubMed]

5. Bylund, G.O.; Persson, B.C.; Lundberg, L.A.; Wikström, P.M. A novel ribosome-associated protein is important for efficient translation in Escherichia coli. J. Bacteriol. 1997, 179, 4567-4574. [CrossRef]

6. Bylund, G.O.; Wipemo, L.C.; Lundberg, L.A.C.; Wikström, P.M. RimM and RbfA Are Essential for Efficient Processing of 16S rRNA in Escherichia coli. J. Bacteriol. 1998, 180, 73-82. [CrossRef]

7. Clatterbuck Soper, S.F.; Dator, R.P.; Limbach, P.A.; Woodson, S.A. In Vivo X-Ray Footprinting of Pre-30S Ribosomes Reveals Chaperone-Dependent Remodeling of Late Assembly Intermediates. Mol. Cell 2013, 52, 506-516. [CrossRef]

8. Lövgren, J.M.; Bylund, G.O.; Srivastava, M.K.; Lundberg, L.A.; Persson, O.P.; Wingsle, G.; Wikström, P.M. The PRC-barrel domain of the ribosome maturation protein RimM mediates binding to ribosomal protein S19 in the 30S ribosomal subunits. RNA 2004, 10, 1798-1812. [CrossRef]

9. Wilson, D.N.; Nierhaus, K.H. The Weird and Wonderful World of Bacterial Ribosome Regulation. Crit. Rev. Biochem. Mol. Biol. 2007, 42, 187-219. [CrossRef]

10. Thurlow, B.; Davis, J.H.; Leong, V.; Moraes, T.F.; Williamson, J.R.; Ortega, J. Binding properties of YjeQ (RsgA), RbfA, RimM and Era to assembly intermediates of the $30 \mathrm{~S}$ subunit. Nucleic Acids Res. 2016, 44, 9918-9932. [CrossRef]

11. Leong, V.; Kent, M.; Jomaa, A.; Ortega, J. Escherichia coli rimM and yjeQ null strains accumulate immature $30 \mathrm{~S}$ subunits of similar structure and protein complement. RNA 2013, 19, 789-802. [CrossRef]

12. Bunner, A.E.; Nord, S.; Wikström, P.M.; Williamson, J.R. The Effect of Ribosome Assembly Cofactors on In Vitro 30 S Subunit Reconstitution. J. Mol. Biol. 2010, 398, 1-7. [CrossRef]

13. Suzuki, S.; Tatsuguchi, A.; Matsumoto, E.; Kawazoe, M.; Kaminishi, T.; Shirouzu, M.; Muto, Y.; Takemoto, C.; Yokoyama, S. Structural Characterization of the Ribosome Maturation Protein, RimM. J. Bacteriol. 2007, 189, 6397-6406. [CrossRef]

14. Anantharaman, V.; Aravind, L. The PRC-barrel: A widespread, conserved domain shared by photosynthetic reaction center subunits and proteins of RNA metabolism. Genome Biol. 2002, 3, RESEARCH0061. [CrossRef]

15. Hollingworth, D.; Candel, A.M.; Nicastro, G.; Martin, S.R.; Briata, P.; Gherzi, R.; Ramos, A. KH domains with impaired nucleic acid binding as a tool for functional analysis. Nucleic Acids Res. 2012, 40, 6873-6886. [CrossRef]

16. Fathir, I.; Mori, T.; Nogi, T.; Kobayashi, M.; Miki, K.; Nozawa, T. Structure of the H subunit of the photosynthetic reaction center from the thermophilic purple sulfur bacterium, Thermochromatium tepidum. Eur. J. Biochem. 2001, 268, 2652-2657. [CrossRef]

17. Mistry, J.; Chuguransky, S.; Williams, L.; Qureshi, M.; Salazar, G.A.; Sonnhammer, E.L.L.; Tosatto, S.C.E.; Paladin, L.; Raj, S.; Richardson, L.J.; et al. Pfam: The protein families database in 2021. Nucleic Acids Res. 2020, 49, D412-D419. [CrossRef]

18. Malakhov, M.P.; Mattern, M.R.; Malakhova, O.A.; Drinker, M.; Weeks, S.D.; Butt, T.R. SUMO fusions and SUMO-specific protease for efficient expression and purification of proteins. J. Struct. Funct. Genom. 2004, 5, 75-86. [CrossRef]

19. Delaglio, F.; Grzesiek, S.; Vuister, G.W.; Zhu, G.; Pfeifer, J.; Bax, A. NMRPipe: A multidimensional spectral processing system based on UNIX pipes. J. Biomol. NMR 1995, 6, 277-293. [CrossRef]

20. Lee, W.; Tonelli, M.; Markley, J.L. NMRFAM-SPARKY: Enhanced software for biomolecular NMR spectroscopy. Bioinformatics 2014, 31, 1325-1327. [CrossRef]

21. Zhang, H.; Guo, C.; Lin, D. ${ }^{1} \mathrm{H},{ }^{13} \mathrm{C},{ }^{15} \mathrm{~N}$ backbone and side-chain NMR assignments of the C-terminal Domain of Mycobacterium Tuberculosis Ribosome Maturation Factor RimM. Biomol. NMR. Assign. under review.

22. Rieping, W.; Habeck, M.; Bardiaux, B.; Bernard, A.; Malliavin, T.E.; Nilges, M. ARIA2: Automated NOE assignment and data integration in NMR structure calculation. Bioinformatics 2006, 23, 381-382. [CrossRef]

23. Shen, Y.; Bax, A. Protein backbone and sidechain torsion angles predicted from NMR chemical shifts using artificial neural networks. J. Biomol. NMR 2013, 56, 227-241. [CrossRef]

24. Laskowski, R.A.; MacArthur, M.W.; Moss, D.S.; Thornton, J.M. PROCHECK: A program to check the stereochemical quality of protein structures. J. Appl. Crystallogr. 1993, 26, 283-291. [CrossRef]

25. Schrödinger LLC. The PyMOL Molecular Graphics System, Version 2.4; Schrödinger LLC: New York, NY, USA, 2020.

26. Cole, R.; Loria, J.P. FAST-Modelfree: A program for rapid automated analysis of solution NMR spin-relaxation data. J. Biomol. NMR 2003, 26, 203-213. [CrossRef]

27. Dosset, P.; Hus, J.-C.; Blackledge, M.; Marion, D. Efficient analysis of macromolecular rotational diffusion from heteronuclear relaxation data. J. Biomol. NMR 2000, 16, 23-28. [CrossRef]

28. Williamson, M.P. Using chemical shift perturbation to characterise ligand binding. Prog. Nucl. Magn. Reson. Spectrosc. 2013, 73, 1-16. [CrossRef] 
29. Larkin, M.A.; Blackshields, G.; Brown, N.P.; Chenna, R.; McGettigan, P.A.; McWilliam, H.; Valentin, F.; Wallace, I.M.; Wilm, A.; Lopez, R.; et al. Clustal W and Clustal X version 2.0. Bioinformatics 2007, 23, 2947-2948. [CrossRef]

30. Robert, X.; Gouet, P. Deciphering key features in protein structures with the new ENDscript server. Nucleic Acids Res. 2014, 42, W320-W324. [CrossRef]

31. Frishman, D.; Argos, P. Knowledge-based protein secondary structure assignment. Proteins: Struct. Funct. Bioinf. 1995, 23, 566-579. [CrossRef]

32. Tian, C.; Kasavajhala, K.; Belfon, K.A.A.; Raguette, L.; Huang, H.; Migues, A.N.; Bickel, J.; Wang, Y.; Pincay, J.; Wu, Q.; et al. ff19SB: Amino-Acid-Specific Protein Backbone Parameters Trained against Quantum Mechanics Energy Surfaces in Solution. J. Chem. Theory Comput. 2020, 16, 528-552. [CrossRef]

33. Case, D.A.; Belfon, K.; Ben-Shalom, I.Y.; Brozell, S.R.; Cerutti, D.S.; Cheatham, T.E., III; Cruzeiro, V.W.D.; Darden, T.A.; Duke, R.E.; Giambasu, G.; et al. AMBER 2020; University of California Press: San Francisco, CA, USA, 2020.

34. Izadi, S.; Anandakrishnan, R.; Onufriev, A.V. Building Water Models: A Different Approach. J. Phys. Chem. Lett. 2014, 5, 3863-3871. [CrossRef] [PubMed]

35. Ryckaert, J.-P.; Ciccotti, G.; Berendsen, H.J.C. Numerical integration of the cartesian equations of motion of a system with constraints: Molecular dynamics of n-alkanes. J. Comput. Phys. 1977, 23, 327-341. [CrossRef]

36. Roe, D.R.; Cheatham, T.E. PTRAJ and CPPTRAJ: Software for Processing and Analysis of Molecular Dynamics Trajectory Data. J. Chem. Theory Comput. 2013, 9, 3084-3095. [CrossRef] [PubMed]

37. Kabsch, W.; Sander, C. Dictionary of protein secondary structure: Pattern recognition of hydrogen-bonded and geometrical features. Biopolymers 1983, 22, 2577-2637. [CrossRef]

38. Wang, C.; Bradley, P.; Baker, D. Protein-Protein Docking with Backbone Flexibility. J. Mol. Biol. 2007, 373, 503-519. [CrossRef]

39. Marze, N.A.; Roy Burman, S.S.; Sheffler, W.; Gray, J.J. Efficient flexible backbone protein-protein docking for challenging targets. Bioinformatics 2018, 34, 3461-3469. [CrossRef]

40. Waterhouse, A.; Bertoni, M.; Bienert, S.; Studer, G.; Tauriello, G.; Gumienny, R.; Heer, F.T.; de Beer, T.A.P.; Rempfer, C.; Bordoli, L.; et al. SWISS-MODEL: Homology modelling of protein structures and complexes. Nucleic Acids Res. 2018, 46, W296-W303. [CrossRef]

41. Kumar, A.; Wilderman, P.R.; Tu, C.; Shen, S.; Qu, J.; Estrada, D.F. Evidence of Allosteric Coupling between Substrate Binding and Adx Recognition in the Vitamin D Carbon-24 Hydroxylase CYP24A1. Biochemistry 2020, 59, 1537-1548. [CrossRef]

42. Clore, G.M.; Szabo, A.; Bax, A.; Kay, L.E.; Driscoll, P.C.; Gronenborn, A.M. Deviations from the simple two-parameter model-free approach to the interpretation of nitrogen-15 nuclear magnetic relaxation of proteins. J. Am. Chem. Soc. 1990, 112, 4989-4991. [CrossRef]

43. Rossi, P.; Swapna, G.V.T.; Huang, Y.J.; Aramini, J.M.; Anklin, C.; Conover, K.; Hamilton, K.; Xiao, R.; Acton, T.B.; Ertekin, A.; et al. A microscale protein NMR sample screening pipeline. J. Biomol. NMR 2010, 46, 11-22. [CrossRef]

44. Lipari, G.; Szabo, A. Model-free approach to the interpretation of nuclear magnetic resonance relaxation in macromolecules. 1. Theory and range of validity. J. Am. Chem. Soc. 1982, 104, 4546-4559. [CrossRef]

45. Lipari, G.; Szabo, A. Model-free approach to the interpretation of nuclear magnetic resonance relaxation in macromolecules. 2. Analysis of experimental results. J. Am. Chem. Soc. 1982, 104, 4559-4570. [CrossRef]

46. Yang, K.; Chang, J.-Y.; Cui, Z.; Li, X.; Meng, R.; Duan, L.; Thongchol, J.; Jakana, J.; Huwe, C.M.; Sacchettini, J.C.; et al. Structural insights into species-specific features of the ribosome from the human pathogen Mycobacterium tuberculosis. Nucleic Acids Res. 2017, 45, 10884-10894. [CrossRef]

47. Maguire, B.A. Inhibition of Bacterial Ribosome Assembly: A Suitable Drug Target? Microbiol. Mol. Biol. Rev. 2009, 73, 22-35. [CrossRef]

48. Kumar, A.; Estrada, D.F. Specificity of the Redox Complex between Cytochrome P450 24A1 and Adrenodoxin Relies on Carbon-25 Hydroxylation of Vitamin-D Substrate. Drug Metab. Dispos. 2019, 47, 974-982. [CrossRef]

49. Borrel, A.; Regad, L.; Xhaard, H.; Petitjean, M.; Camproux, A.-C. PockDrug: A Model for Predicting Pocket Druggability That Overcomes Pocket Estimation Uncertainties. J. Chem. Inf. Model. 2015, 55, 882-895. [CrossRef] 